Pacific Journal of Mathematics

AN INDIRECT SUFFICIENCY PROOF FOR PROBLEMS WITH

I. Bert Russ 


\section{AN INDIRECT SUFFICIENCY PROOF FOR PROBLEMS WITH BOUNDED STATE VARIABLES}

\section{Bert RussAK}

A set of sufficient conditions is obtained for problems involving constraints of the form $\psi^{\alpha}(t, x) \leqq 0 \alpha=1, \cdots, m$. The method of proof is indirect. It is shown by essentially strengthening the first and second order necessary results previously obtained by the author for problems of this type, that a proper strong relative minimum is obtained.

1. Introduction. Consider the class of arcs

$$
\begin{array}{lll}
a: x^{l}(t), & p^{k}(t), \quad b^{\sigma}, & t^{0} \leqq t \leqq t^{1} \\
i=1, \cdots, N & k=1, \cdots, K & \sigma=1, \cdots, r
\end{array}
$$

where ${ }^{1}$ elements $(t, x(t), p(t))$ and $b$ lie respectively in open sets $R$ in txp space and $B$ in $b$ space. The terms $x^{\prime}$ are called state variables while the terms $p^{k}, b^{\sigma}$ are called control variables and control parameters respectively. We require these arcs to satisfy the constraints

$$
\begin{aligned}
& (1-1) \dot{x}^{\prime}(t)=f^{i}(t, x(t), p(t)), \quad i=1, \cdots, N \quad \text { a.e. in }\left[t^{0}, t^{1}\right] \\
& (1-2) \quad \psi^{\alpha}(t, x(t)) \leqq 0 \quad \alpha=1, \cdots, m \quad\left[t^{0}, t^{1}\right] \\
& (1-3) \quad I_{\gamma}(a) \leqq 0 \quad \gamma=1, \cdots, p^{\prime} \quad I_{\gamma}(a)=0 \quad \gamma=p^{\prime}+1, \cdots, p \\
& (1-4) \quad x^{\prime}\left(t^{s}\right)=X^{i s}(b) \quad s=0,1 \\
& (1-5) \text { where } I_{\gamma}(a)=g_{\gamma}(b)+\int_{t^{0}}^{t^{1}} L_{\gamma}(t, x(t), p(t)) d t \quad \gamma=1, \cdots, p
\end{aligned}
$$

and are interested in minimizing the functional

$$
I_{0}(a)=g_{0}(b)+\int_{t^{0}}^{t^{1}} L_{0}(t, x(t), p(t)) d t
$$

Let $C$ be the class of arcs described above with $x(t)$ absolutely continuous, $p(t), f(t, x(t), p(t))$ and $L_{\gamma}(t, x(t), p(t)) \gamma=0,1, \cdots, p$ integrable on $\left[t^{0}, t^{1}\right]$. It is desired to minimize $I_{0}(\boldsymbol{a})$ on the class $C$.

' Unless otherwise noted, the indices $i, k, \sigma, \alpha$ will have the respective ranges $i=1, \cdots, N$, $k=1, \cdots, K, \sigma=1, \cdots, r$ and $\alpha=1, \cdots, m$. 
In [2] and [3] the author establishes first order necessary conditions for this problem. By essentially strengthening those conditions, and extending a technique devised originally by Hestenes and used by Pennisi in [1], a set of sufficient conditions for a proper strong relative minimum is obtained without the use of field theory and an invariant integral.

2. Assumptions. Using the problem defined above, the functions will be assumed to possess the following continuity properties; the functions $\psi^{\alpha}$ will be class $C^{3}$ while the functions $f^{i}, X^{i s}, g_{\gamma}, L_{\gamma}, L_{0}$, and $g_{0}$ will be of class $C^{2}$. Also, an arc will be called admissible if it in the class $C$.

Next, define the functions ${ }^{2} \phi^{\alpha}=\psi_{t}^{\alpha}+\psi_{x^{\prime}}^{\alpha} f^{\prime} \alpha=1, \cdots, m$. These functions act as derivatives of $\psi^{\alpha}$ along admissible arcs. Also define the set $R_{0}$ of points (txp) in $R$ satisfying

$$
\psi^{\alpha} \leqq 0
$$

$$
\phi^{\alpha} \geqq 0 \text { for all } \alpha \text { with } \psi^{\alpha}=0 \text { or } \phi^{\alpha} \leqq 0 \text { for all } \alpha \text { with } \psi^{\alpha}=0 \text {. }
$$

We shall be concerned with a particular admissible arc $\boldsymbol{a}_{0}$

$$
a_{0}: x_{0}^{i}(t), \quad p_{0}^{k}(t), \quad b_{0}^{\sigma}, \quad t^{0} \leqq t \leqq t^{1}
$$

and shall make some assumptions about the arc $\boldsymbol{a}_{0}$. In order to state these we first make the following definitions:

The set $S^{\alpha}$ is the set of $t$ such that $\psi^{\alpha}\left(t, x_{0}(t)\right)=0$.

For each $t$, the symbol $\Gamma(t)$ denotes the set of indices $\alpha$ such that $\psi^{\alpha}\left(t, x_{0}(t)\right)=0$.

We will have need to talk of the quantities $z_{t}(t), \mu_{\alpha}(t), \lambda_{\rho}, K^{\alpha}$ where $z_{l}(t)$ are of class $C^{1}, \mu_{\alpha}(t)$ are absolutely continuous functions with continuous derivatives $\dot{\mu}_{\alpha}(t)$ and $K^{\alpha}, \lambda_{\rho}(\rho=1, \cdots, p+N)$ are constants.

For each $t$, the set $\Delta(t)$ is the set of $\alpha$ indices such that $\dot{\mu}_{\alpha}(t) \neq 0$.

The functions $\bar{\psi}^{\alpha}$ and $\bar{\phi}^{\alpha}$ are defined as

$$
\bar{\psi}^{\alpha} \equiv \psi^{\alpha} /\left[1+\left(\psi^{\alpha}\right)^{2}\right]^{\frac{1}{2}} \quad \bar{\phi}^{\alpha} \equiv \phi^{\alpha} /\left[1+\left(\phi^{\alpha}\right)^{2}\right]^{\frac{1}{2}}
$$

Our assumptions concerning $\boldsymbol{a}_{0}$ are as follows:

(i) $p_{0}(t)$ is continuous in $\left[t^{0}, t^{1}\right]$.

(ii) For each $t$ the set $\Gamma(t)-\Delta(t)$ contains at most one index.

(iii) The matrix $\left(\phi_{p^{\alpha}}^{\alpha}\right)$ has rank $m$ along $\boldsymbol{a}_{0}$.

(iv) There exist the quantities $z_{i}(t), \mu_{\alpha}(t), K^{\alpha}, \lambda_{\rho}$. $(\rho=1, \cdots, p+N)$ as referred to above, satisfying

${ }^{2}$ For a function $M(t, x, p, b)$ the notations $M_{x^{\prime}}, M_{p^{k}}, M_{b} \sigma, M_{t}$ will denote first partial derivatives with respect to the indicated variable. Also repeated indices will be summed. 
$(4-1)$

$$
\begin{gathered}
\lambda_{\gamma} \geqq 0 \quad \text { with } \quad \lambda_{\gamma}=0 \quad \text { if } \quad I_{\gamma}\left(a_{0}\right)<0 \quad 1 \leqq \gamma \leqq p^{\prime}, \\
K^{\alpha} \geqq 0, \quad \lambda_{p+i}=K^{\alpha} \psi_{x^{\prime}}^{\alpha}\left(t^{0}\right) \\
\mu_{\alpha}\left(t^{1}\right)=0 \quad \text { if } \quad \psi^{\alpha}\left(t^{1}\right)<0 \\
\mu_{\alpha}\left(t^{0}\right) \leqq K^{\alpha} \quad \text { with } \quad \mu_{\alpha}\left(t^{0}\right)=K^{\alpha} \quad \text { if } \quad \psi^{\alpha}\left(t^{0}\right)<0
\end{gathered}
$$

and also such that the functions $\mu_{\alpha}(t)$ are nonincreasing nonnegative functions which are constant on intervals where $\psi^{3}(t)<0$. Notice that this last statement means

$$
\int_{t^{0}}^{t^{1}} \dot{\mu}_{\alpha}(t) \psi^{\alpha}(t) d t=0
$$

Using the terms of (iv) define the functions

$$
\begin{aligned}
& \mathscr{G}(b) \equiv g_{0}(b)+\lambda_{\gamma} g_{\gamma}(b)+\lambda_{p+1} X^{i 0}(b) \quad \gamma=1, \cdots, p \\
& H(t, x, p, z(t), \mu(t)) \equiv z_{\imath}(t) f^{i}-L_{0}-\lambda_{\gamma} L_{\gamma}-\mu_{\alpha}(t) \phi^{\alpha} \\
& G(t, x, p, z(t), \mu(t)) \equiv-H(t, x, p, z(t), \mu(t))-\dot{z}_{l}(t) x^{i}
\end{aligned}
$$

and the Weierstrass $E$ function for $G$,

(7) $E_{G}(t, x, p, q, z(t), \mu(t)) \equiv G(t, x, q, z(t), \mu(t))-G(t, x, p, z(t), \mu(t))$

$$
-\left(q^{k}-p^{k}\right) G_{p^{k}}(t, x, p, z(t), \mu(t)) \text {. }
$$

Then the following statements are true:

(va) The arc $\boldsymbol{a}_{0}$ satisfies the transversality relation

$$
d \mathscr{G}+\left[z_{i}\left(t^{s}\right) d X^{i s}\right]_{s=0}^{s=1}=0
$$

(where e.g. $d \mathscr{G}$ means $\mathscr{G}_{b^{\sigma}}\left(b_{0}\right) d b^{\sigma}$ ) for arbitrary vectors $d b$.

(vb) The relations

$$
\dot{z}_{l}=-H_{x^{i}} \quad \dot{x}^{\imath}=H_{z^{\prime}} \quad H_{p^{k}}=0
$$

hold along $\boldsymbol{a}_{0}$. Note that (9) implies that

$$
G_{x^{\prime}}=0 \quad G_{p^{k}}=0
$$

along $\boldsymbol{a}_{0}$.

${ }^{3}$ Henceforth unless otherwise stated, a function $M(t, x, p, b)$ evaluated along $a_{0}$ at $\left(t, x_{0}(t), p_{0}(t), b_{0}\right)$ will be denoted by $M(t)$ or if just a function of $b$, it will be denoted by $M\left(b_{0}\right)$ or just M. 
(vc) There is a positive constant $b$ and a neighborhood $D_{1}$ of $a_{0}$ relative to $R_{0}$ such that ${ }^{4}$

$$
\begin{aligned}
& E_{G}(t, x, p, q, z(t), \mu(t))+\dot{\mu}_{\alpha}(t) \psi^{\alpha}(t, x) \\
& \quad \geqq b\left[E_{L}(p, q)+\max \left(\dot{\mu}_{\alpha}(t) \bar{\psi}^{\alpha}(t, x),\left|\dot{\mu}_{\alpha}(t) \bar{\phi}^{\alpha}(t, x, q)\right|\right)\right]
\end{aligned}
$$

and with $E_{\gamma}$ as the $E$ function for $L_{\gamma}$, also

(12) $E_{G}(t, x, p, q, z(t), \mu(t))+\dot{\mu}_{\alpha}(t) \psi^{\alpha}(t, x) \geqq b\left|E_{\gamma}(t, x, p, q)\right| \gamma=1, \cdots, p$

when $(t, x, p)$ is in $D_{1},(t, x, q)$ is in $R_{0}$ and $\phi^{\alpha}(t, x, p)=0$ if $\dot{\mu}_{\alpha}(t) \neq 0$. The function $E_{L}(p, q)$ is the $E$ function for the function

$$
L(p) \equiv\left(1+p^{k} p^{k}\right)^{\frac{1}{2}}
$$

which is the integrand of the length integral. Thus

$$
E_{L}(p, q) \equiv L(q)-L(p)-\left(q^{k}-p^{k}\right) L_{p^{k}}(p)
$$

which is the same as

$$
E_{L}(p, q)=L(q)-\frac{1+\left(p^{k} q^{k}\right)}{L(p)}
$$

(vi) There is a neighborhood of $a_{0}$ in $t x$ space and a positive constant $\rho$ such that the Lipschitz condition

$$
\mid f(t, x, p)-f(t, z, q)) \mid<\rho\left[|x-z|^{2}+|p-q|^{2}\right]^{\frac{1}{2}}
$$

holds for all points $(t, x, p)$ and $(t, z, q)$ in that neighborhood.

We note that the majority of these assumptions about $\boldsymbol{a}_{0}$ are either just the necessary conditions for a solution to our problem or the assumptions used in proving these necessary conditions as shown in [2] and [3].

In particular, the only assumptions listed which do not come under those headings ${ }^{5}$ are:

(a) the existence of $\dot{\mu}_{\alpha}(t)$ on $S^{\alpha}$ sets, and

(b) the assumptions (ii), (vc) and (vi).

${ }^{4}$ We shall often omit the argument $t$ when referring to the functions $\mu_{\alpha}(t)$ and $\dot{\mu}_{\alpha}(t)$ of (4). However we shall always understand the terms $\mu_{\alpha}$ and $\dot{\mu}_{\alpha}$ to refer to those functions.

5 A note soon to appear will modify the results of [3] to include the last stated property in (4-1) as a necessary condition. 


\section{Statement of the main result and definition of an} admissible variation. We next define what we shall mean by an admissible variation of the $\operatorname{arc} \boldsymbol{a}_{0}$.

A set of functions

$$
\delta a: \delta x^{i}(t), \quad \delta p^{k}(t), \quad \delta b^{\sigma}, \quad t^{0} \leqq t \leqq t^{1}
$$

with $\delta x(t)$ absolutely continuous and $\delta p(t), \delta \dot{x}(t)$ square integrable on $\left[t^{0}, t^{1}\right]$ will be called a variation of the arc $a_{0}$ and for any function $M(t, x, p, b)$ which possesses first partial derivatives along $a_{0}$ we call

$$
\delta M(t)=M_{x^{i}}(t) \delta x^{i}(t)+M_{p^{k}}(t) \delta p^{k}(t)+M_{b^{\sigma}}(t) \delta b^{\sigma}
$$

the variation of $M$ along $a_{0}$ due to the variation $\delta a$.

Next define the functionals of any arc $\boldsymbol{a}$ as:

$$
\begin{aligned}
& J_{\gamma}(a)=I_{\gamma}(a), \quad \gamma=0,1, \cdots, p \\
& \left.\begin{array}{l}
J_{p+\imath}(a)=x^{\prime}\left(t^{1}\right)-X^{i 1}(b) \\
J_{p+N+i}(a)=X^{i 0}(b)-x^{i}\left(t^{0}\right)
\end{array}\right\} \quad i=1, \cdots, N,
\end{aligned}
$$

and the variations of these functionals due to the variation $\delta a$ as:

$$
J_{\gamma}^{\prime}\left(a_{0}, \delta a\right)=\delta g_{\gamma}\left(b_{0}\right)+\int_{t^{0}}^{t^{1}} \delta L_{\gamma}(t) d t \quad \gamma=0,1, \cdots, p
$$

$$
\left.\begin{array}{l}
J_{p+i}^{\prime}\left(a_{0}, \delta \boldsymbol{a}\right)=\delta x^{i}\left(t^{1}\right)-\delta X^{i 1}\left(b_{0}\right) \\
J_{p+i}^{\prime}\left(\boldsymbol{a}_{0}, \delta \boldsymbol{a}\right)=\delta X^{i 0}\left(b_{0}\right)-\delta x^{i}\left(t^{0}\right)
\end{array}\right\} \quad i=1, \cdots, N .
$$

Also let $\gamma_{k}$ be those indices $1 \leqq \gamma \leqq p^{\prime}$ for which

$$
I_{\gamma}\left(a_{0}\right)<0 \quad \gamma=\gamma_{k} .
$$

Then, we know by (4) that

$$
\lambda_{\gamma_{k}}=0 \text {. }
$$

With these definitions in mind, we call a variation admissible if the following are true:

$$
\delta \dot{x}^{i}(t)=\delta f^{i}(t) \quad \text { a.e. on }\left[t^{0}, t^{1}\right]
$$

$$
\delta \psi^{\alpha}(t) \leqq 0 \quad \text { on } \quad S^{\alpha}
$$




$$
\delta \psi^{\alpha}\left(t^{1}\right)=0 \quad \text { if } \quad \mu_{\alpha}\left(t^{1}\right) \neq 0
$$

$$
\dot{\mu}_{\alpha}(t) \delta \psi^{\alpha}(t)=0
$$

a.e. on $\left[t^{0}, t^{1}\right]$

( $\alpha$ not summed)

$$
\delta \psi^{\alpha}\left(t^{0}\right)=0 \quad \text { if } \quad \mu_{\alpha}\left(t^{0}\right) \neq K^{\alpha}
$$

$$
J_{\gamma}^{\prime}\left(a_{0}, \delta a\right)=0 \quad \text { if } \quad \lambda_{\gamma} \neq 0 \quad 1 \leqq \gamma \leqq p^{\prime}
$$

$$
J_{\gamma}^{\prime}\left(a_{0}, \delta a\right) \leqq 0 \quad \text { if } \quad \lambda_{\gamma}=0 \quad \gamma \neq \gamma_{k}
$$

$$
J_{\rho}^{\prime}\left(a_{0}, \delta a\right)=0 \quad p^{\prime}<\rho \leqq p+2 N .
$$

For each such admissible variation the second variation

$$
\begin{aligned}
& J_{2}\left(a_{0}, \delta a\right)= {\left[\left(z^{i}\left(t^{s}\right) X_{b^{\sigma} b^{\tau}}^{i s}\right)_{s=0}^{s=1}+\mathscr{G}_{b^{\sigma} b^{\tau}}+K^{\alpha} \psi_{x^{i} x}^{\alpha_{x}}\left(t^{0}\right) X_{b^{\sigma}}^{i 0} X_{b^{\tau}}^{j 0}\right] \delta b^{\sigma} \delta b^{\tau} } \\
&+ \int_{t^{0}}^{t^{1}}\left[G_{x^{i} x^{\prime}} \delta x^{i} \delta x^{j}+2 G_{x^{i} p^{k}} \delta x^{i} \delta p^{k}+G_{p^{h} p^{k}} \delta p^{h} \delta p^{k}\right] d t \\
& i, j=1, \cdots, N \quad \sigma, \tau=1, \cdots, r \quad \alpha=1, \cdots, m \quad h, k=1, \cdots, K,
\end{aligned}
$$

(where $\mathscr{G}, G$ are the functions of (5) and (6-2) respectively, and $K^{\alpha}$ are the constants referred to in assumption (iv)) is well defined.

The Theorem to be proven in this paper is:

THEOREM 3.1. Let $\boldsymbol{a}_{0}$ be an admissible arc which satisfies assumptions (i) through (vi) and suppose that $J_{2}\left(a_{0}, \delta a\right)>0$ for every non-null admissible variation. Then there is a neighborhood $F$ of $a_{0}$ in $t x b$ space such that the inequality $I_{0}(a)>I_{0}\left(a_{0}\right)$ holds for every admissible arc $a$ in $F$ which is different from $\boldsymbol{a}_{0}$.

It is noted that [2] proves as a second order necessary condition that $J_{2}\left(a_{0}, \delta a\right) \geqq 0$ for all such admissible variations $\delta a$ as described above ${ }^{6}$. Thus the hypotheses of the theorem is only a strengthened necessary condition.

Henceforth unless otherwise stated, our arc $a_{0}$ will be assumed to satisfy the conditions (i) through (vi) and we shall not explicitly state this each time we refer to $a_{0}$.

4. Convergent sequences of admissible arcs. We proceed in a manner similar to [1]. Consider a sequence ${ }^{7}\left\{\boldsymbol{a}_{k}\right\}$ of admissible arcs which converges uniformly to $a_{0}$ in $t x b$ space.

${ }^{6}$ Actually in [2] the condition (23-5) is replaced by $\delta \psi^{\alpha}\left(t^{0}\right)=0$ if $\mu_{\alpha}\left(t^{0}\right) \neq 0$. However a note to appear soon will extend that proof to the condition which we use.

7 Subscripts attached to the symbols $x, p, b$ will denote association with an arc with that subscript. Thus $p_{k}(t)$ is the value of control along an arc $\boldsymbol{a}_{k}$. 
Using the function $L(p)$ defined in (13) we first define a quantity which will act as part of the square of a norm in arc space. Let

$$
K\left(\boldsymbol{a}, \boldsymbol{a}_{0}\right) \equiv \int_{t^{0}}^{t^{1}}\left[L\left(p(t)-p_{0}(t)\right)-1\right] d t
$$

where $p, p_{0}$ are the respective controls along $\boldsymbol{a}, \boldsymbol{a}_{0}$. Our first major result is

THEOREM 4.1. If $\left\{\boldsymbol{a}_{k}\right\}$ is a sequence of admissible arcs which converges uniformly to $a_{0}$ in txb space and also satisfies $\lim \sup _{k \rightarrow \infty} I_{0}\left(a_{k}\right) \leqq$ $I_{0}\left(\boldsymbol{a}_{0}\right)$, then $\lim _{k \rightarrow \infty} K\left(\boldsymbol{a}_{k}, \boldsymbol{a}_{0}\right)=0$ and there is a subsequence $\left\{\boldsymbol{a}_{k_{r}}\right\}$ of $\left\{\boldsymbol{a}_{k}\right\}$ such that $p_{k_{r}}(t)$ converges to $p_{0}(t)\left[\mathrm{a}\right.$. unif.] on $\left[t^{0}, t^{1}\right]$.

The proof of this theorem will be based on a number of definitions and lemmas which we proceed to list. The first of these is:

LEMMA 4.1. There exist functions $P^{k}(t, x) k=1, \cdots, K$ which are of class $C^{2}$ with respect to $x, C^{0}$ in $t$ and satisfy $\phi^{\alpha}(t, x, P(t, x))-\phi^{\alpha}(t)=0$, $\alpha=1, \cdots, m t^{0} \leqq t \leqq t^{1},\left|x-x_{0}(t)\right|<\xi$ for some positive constant $\xi$. In addition, $P^{k}\left(t, x_{0}(t)\right)=p_{0}^{k}(t)$ on $\left[t^{0}, t^{1}\right]$.

Proof. The proof of this follows from the continuity properties of $\psi^{\alpha}$, the definitions of $\phi^{\alpha}$ and the use of Lemma 10.1 of [3].

Next with the functions $P(t, x)$ of Lemma 4.1 we shall be able to break up the functionals of our problem in a convenient manner.

As a first example of this, we consider the functional $J_{T}(\boldsymbol{a})$ defined by:

$$
J_{T}(\boldsymbol{a})=\left[z^{\prime}\left(t^{s}\right) X^{i s}(b)\right]_{s=0}^{s=1}+g_{0}(b)+\lambda_{\gamma} g_{\gamma}(b)+\int_{t^{0}}^{t^{1}} G(t, x, p) d t .
$$

Notice that by (1-5), (1-6), (6-1), and (6-2) we have (with arguments along $a$ ), that

$$
I_{0}(a)=J_{T}(a)-\lambda_{\gamma} I_{\gamma}(a)-\int_{t^{0}}^{t^{1}} \mu_{\alpha}(t) \phi^{\alpha}(t, x, p) d t .
$$

Next, we write:

$$
\begin{aligned}
(27-1) J_{T}^{*}(\boldsymbol{a})= & {\left[z^{i}\left(t^{s}\right) X^{i s}\left(b_{0}\right)\right]_{s=0}^{s=1}+g_{0}\left(b_{0}\right)+\lambda_{\gamma} g_{\gamma}\left(b_{0}\right) } \\
& +\left[\left[z^{i}\left(t^{s}\right) X_{b^{\sigma}}^{i s}\right]_{s=0}^{s=1}+g_{0_{b^{\sigma}}}+\lambda_{\gamma} g_{\gamma_{b}}\right]\left[b^{\sigma}-b_{0}^{\sigma}\right] \\
& +\int_{t^{0}}^{t^{1}}\left[G(t, x, P)+\left[p^{k}-P^{k}\right] G_{p^{k}}(t, x, P)\right] d t .
\end{aligned}
$$




$$
\begin{aligned}
(27-2) E_{T}^{*}(a)= & \int_{t^{0}}^{t^{1}} E_{G}(t, x, P, p) d t \\
\equiv & \int_{t^{0}}^{t^{1}}\left[G(t, x, p)-G(t, x, P)-\left[p^{k}-P^{k}\right] G_{p^{k}}(t, x, P)\right] d t \\
(27-3) B_{T}^{*}(a)= & z^{t}\left(t^{s}\right)\left[X^{i s}(b)-X^{i s}\left(b_{0}\right)-X_{b^{\sigma}}^{i s}\left[b^{\sigma}-b_{0}^{\sigma}\right]\right]_{s=0}^{s=1}+g_{0}(b)+\lambda_{\gamma} g_{\gamma}(b) \\
& -g_{0}\left(b_{0}\right)-\lambda_{\gamma} g_{\gamma}\left(b_{0}\right)-\left[g_{0_{b^{\sigma}}}+\lambda_{\gamma} g_{\gamma^{\sigma}}\right]\left[b^{\sigma}-b_{0}^{\sigma}\right]
\end{aligned}
$$

where: (i) all derivatives with respect to $b$ are at $b_{0}$; (ii) the arguments $x, p, P, b$ are evaluated along $a$ with $P=P(t, x)$ and (iii) the functions $E_{G}, G$ are the function of (6-2), (7) where for conciseness of notation we have deleted the arguments $z(t), \mu(t)$ but understand them to still be present. This convention in writing the arguments of $E_{G}, G$ will be used throughout this paper.

It is convenient here also to define another functional of an arc $\boldsymbol{a}$.

$$
E_{T}(\boldsymbol{a}) \equiv E_{T}^{*}(\boldsymbol{a})+\int_{t^{0}}^{t^{1}} \dot{\mu}_{\alpha}(t) \psi^{\alpha}(t, x) d t
$$

where $E_{T}^{*}$ is from $(27-2), \dot{\mu}_{\alpha}(t)$ are the derivatives appearing in the assumption (iv) and the arguments $t, x$ are on $\boldsymbol{a}$. With these definitions, we see that

$$
J_{T}(\boldsymbol{a})=J_{T}^{*}(\boldsymbol{a})+B_{T}^{*}(\boldsymbol{a})+E_{T}^{*}(\boldsymbol{a})
$$

Next, let $V(t, x, p)$ be any function of class $C^{2}$. Then we make the following definitions:

(i) For an arc $\boldsymbol{a}$ set

$$
V(a)=\int_{t^{0}}^{t^{1}} V(t, x, p) d t
$$

(where the arguments are along $\boldsymbol{a}$ ) and

(ii) we shall say that $V$ is $E_{T}$ dominated near $a_{0}$ on $R_{0}$ if there is a positive constant $c$ and a neighborhood $R_{1}$ of $a_{0}$ relative to $R_{0}$ such that

$$
E_{G}(t, x, p, q)+\dot{\mu}_{\alpha}(t) \psi^{\alpha}(t, x) \geqq c\left|E_{V}(t, x, p, q)\right|
$$

whenever $t, x, p$ is in $R_{1}, t, x, q$ is in $R_{0}$, and $\phi^{\alpha}(t, x, p)=0$ if $\dot{\mu}_{\alpha}(t) \neq 0$ and where

(30-2) $E_{V}(t, x, p, q) \equiv V(t, x, q)-V(t, x, p)-\left(p^{k}-q^{k}\right) V_{p^{k}}(t, x, p)$

We shall further restrict the neighborhood $R_{1}$ and constant $c$ if necessary so that 


$$
E_{G}(t, x, p, q)+\dot{\mu}_{\alpha}(t) \psi^{\alpha}(t, x) \geqq c E_{L}(p-P(t, x), q-P(t, x))
$$

(where $E_{L}$ is the function introduced in (14)) whenever $t, x, p$ is in $R_{1}$ and $t, x, q$ is in $R_{0}$ and $\phi^{\alpha}(t, x, p)=0$ if $\dot{\mu}_{\alpha}(t) \neq 0$. The proof of this is analagous to [1] Page 30, using our assumption (vc).

We next prove:

LEMMA 4.2. Given $\epsilon^{\prime}>0$ then there is a neighborhood $\mathscr{F}$ of $a_{0}$ in $t x b$ space such that

$$
-\epsilon^{\prime}+\left(1-\epsilon^{\prime}\right) E_{T}(a)<I_{0}(a)-I_{0}\left(a_{0}\right)
$$

for each admissible arc $\boldsymbol{a}$ in $\mathscr{F}$.

Proof. By the definition of the functions $\phi^{\alpha}$ we see that along any admissible arc $\boldsymbol{a}$

$$
\int_{t^{0}}^{t^{1}} \mu_{\alpha} \phi^{\alpha} d t=\int_{t^{0}}^{t^{1}} \frac{d}{d t}\left[\mu_{\alpha} \psi^{\alpha}\right] d t-\int_{t^{0}}^{t^{1}} \dot{\mu}_{\alpha} \psi^{\alpha} d t
$$

with arguments along ${ }^{8} a$. Then by (26-2), (29), (28) and (33), we have for $a$, an admissable arc that

$$
\begin{aligned}
I_{0}(a)= & J_{T}^{*}(a)+\mu_{\alpha}\left(t^{0}\right) \psi^{\alpha}\left(t^{0}, x\left(t^{0}\right)\right)+E_{T}(a)+B_{T}^{*}(a)-\lambda_{\gamma} I_{\gamma}(a) \\
& -\mu_{\alpha}\left(t^{1}\right) \psi^{\alpha}\left(t^{1}, x\left(t^{1}\right)\right)
\end{aligned}
$$

(where $x\left(t^{0}\right), x\left(t^{1}\right)$ are along $a$ ).

Then with $\Delta$ denoting ${ }^{9}$ the change in a quantity evaluated from $a_{0}$ to $a$, so that e.g.

$$
\Delta I_{0}(\boldsymbol{a}) \equiv I_{0}(\boldsymbol{a})-I_{0}\left(\boldsymbol{a}_{0}\right)
$$

we have

$$
\text { (36) } \begin{aligned}
\Delta I_{0}(\boldsymbol{a})= & \Delta J_{T}^{*}(\boldsymbol{a})+\mu_{\alpha}\left(t^{0}\right) \Delta \psi^{\alpha}\left(t^{0}, x\left(t^{0}\right)\right)+E_{T}(\boldsymbol{a})+B_{T}^{*}(\boldsymbol{a})-\lambda_{\gamma} \Delta I_{\gamma}(\boldsymbol{a}) \\
& -\mu_{\alpha}\left(t^{1}\right) \Delta \psi^{\alpha}\left(t^{1}, x\left(t^{1}\right)\right)
\end{aligned}
$$

where in (36) we have recognized, because of (4-2) together with the

${ }^{8}$ We shall often omit arguments in this fashion whenever the context makes clear what those arguments are.

${ }^{9}$ Henceforth we shall use this notation frequently, thus as another example $\Delta x_{\gamma}(t)$ will mean $x_{\gamma}(t)-x_{0}(t)$ where $x_{\gamma}(t)$ is the value of state on an arc $a_{\gamma}$. 
definition of $E_{T}^{*}$ in (27-2) and the construction of the functions $P$ of Lemma 4.1 , that $E_{T}\left(a_{0}\right)=0$ and also, by $(27-3)$, that $B_{T}^{*}\left(a_{0}\right)=0$.

Now in (31) set $p^{k}=P^{k}$ so that

$$
\begin{aligned}
c[L(q-P(t, x))-1]= & c E_{L}(0, q-P(t, x)) \leqq E_{G}(t, x, P(t, x), q) \\
& +\dot{\mu}_{\alpha}(t) \psi^{\alpha}(t, x)
\end{aligned}
$$

which is true for $t, x$ in some neighborhood $\overline{\mathscr{F}}$ (in $t x$ space) reduced if necessary from the projection of $R_{1}$ of (31) and also for $t, x, q$ in $R_{0}$.

Next with $p(t)$ having the value associated with the arc $a$, set $q$ equal to $p(t)$ so that we get

$$
\begin{aligned}
|p(t)-P(t, x)| & <L(p(t)-P(t, x)) \\
& \leqq c^{-1}\left[1+E_{G}(t, x, P(t, x), p(t))+\dot{\mu}_{\alpha}(t) \psi^{\alpha}(t, x)\right]
\end{aligned}
$$

for $t, x$ in the neighborhood $\overline{\mathscr{F}}$ of $(37)$, where in (38) we have increased $c^{-1}$ to be greater than 1 if it was not already so.

Now consider the integral part of $\Delta J_{T}^{*}(\boldsymbol{a})$, that is the terms

$$
\int_{t^{0}}^{t^{1}}\left[G(t, x, P(t, x))-G(t)+\left(p^{k}-P^{k}(t, x)\right) G_{p^{k}}(t, x, P(t, x))\right] d t
$$

where $x, p$ are $x(t), p(t)$ of $a$. Since $G$ satisfies (10), then we can find a neighborhood $\overline{\mathscr{F}}$ of $a_{0}$ in $t x$ space such that

$$
\left|G_{p^{k}}(t, x, P(t, x))\right|<\epsilon
$$

for $t, x$ in $\overline{\bar{F}}$. Then by (38) and (40) we have with $\mathscr{F}$ as the intersection of $\overline{\mathscr{F}}$ and $\overline{\mathscr{F}}$ that if $\boldsymbol{a}$ is in $\mathscr{F}$

$$
\left|\int_{t^{0}}^{t^{1}}\left(p^{k}-P^{k}(t, x)\right) G_{p^{k}}(t, x, P(t, x)) d t\right|<\epsilon c^{-1}\left[t^{1}-t^{0}+E_{T}(a)\right] .
$$

Also with $\mathscr{F}$ small enough we will have

$$
\mid G(t, x, P(t, x)-G(t) \mid<\epsilon
$$

for $t, x$ in $\mathscr{F}$.

Thus by (41) and (42) we have that for $\boldsymbol{a}$ an admissible arc in $\mathscr{F}$, then

$$
\begin{gathered}
\int_{t^{0}}^{t^{\prime}}\left[G(t, x, P(t, x))-G(t)+\left(p^{k}-P^{k}(t, x)\right) G_{p^{k}}(t, x, P(t, x))\right] d t \\
<\epsilon\left[t^{1}-t^{0}\right]+\epsilon c^{-1}\left[t^{1}-t^{0}+E_{T}(a)\right]
\end{gathered}
$$


Now except for the quantities $\lambda_{\gamma} \Delta I_{\gamma}$ and $E_{T}(a)$, all other terms on the right hand side of (36) depend solely on $t x b$ values and vanish on $a_{0}$. Then by considering $\mathscr{F}$ as a neighborhood in txb space and making it small enough, we can make the sum of all of these quantities less than $\epsilon$ and then by (43) and (37) achieve for an admissible arc $\boldsymbol{a}$ in $\mathscr{F}$,

$$
\left|\Delta I_{0}(a)+\lambda_{\gamma} \Delta I_{\gamma}(a)-E_{T}(a)\right|<\epsilon\left[t^{1}-t^{0}+1\right]+\epsilon c^{-1}\left[t^{1}-t^{0}+E_{T}(a)\right] .
$$

Inequality (44) implies that

$$
E_{T}(a)-\lambda_{\gamma} \Delta I_{\gamma}(a)-\epsilon\left[t^{1}-t^{0}+1\right]-\epsilon c^{-1}\left[t^{1}-t^{0}+E_{T}(a)\right]<\Delta I_{0}(a) .
$$

Now by the admissibility of $a$ and the nonnegativity of $\lambda_{\gamma}$ (see (4)), we see that

$$
-\lambda_{\gamma} \Delta I_{\gamma}(a) \geqq 0
$$

then (46) implies that for $\boldsymbol{a}$ admissible and in $\mathscr{F}$

$$
-\epsilon\left[\left(1+c^{-1}\right)\left(t^{1}-t^{0}\right)+1\right]+\left(1-\epsilon c^{-1}\right) E_{T}(a)<\Delta I_{0}(a) .
$$

Now select $\epsilon$ such that

$$
\epsilon\left[\left(1+c^{-1}\right)\left(t^{1}-t^{0}\right)+1\right]<\epsilon^{\prime} \text { and } \quad \epsilon c^{-1}<\epsilon^{\prime}
$$

so that our lemma is proven.

Now with $V(\boldsymbol{a})$ as the functional defined below (29) we prove:

LEMMA 4.3. If $V(t, x, p)$ satisfies

$$
V_{p^{k}}\left(t, x_{0}(t), p_{0}(t)\right)=0 \quad t^{0} \leqq t \leqq t^{1}
$$

and if $V$ is $E_{T}$ dominated near $a_{0}$ on $R_{0}$ with constant $c$, then for each $\epsilon^{\prime}>0$ there is a neighborhood $\mathscr{K}$ of $a_{0}$ in tx space such that

$$
\left|V(\boldsymbol{a})-V\left(\boldsymbol{a}_{0}\right)\right|<\boldsymbol{\epsilon}^{\prime}+\left(c^{-1}+\boldsymbol{\epsilon}^{\prime}\right) E_{T}(\boldsymbol{a}) \text {. }
$$

Proof. Set

(50)

$$
V^{*}(a)=\int_{t^{0}}^{t^{1}}\left[V(t, x, P(t, x))+\left(p^{k}-P^{k}(t, x)\right) V_{p^{k}}(t, x, P(t, x))\right] d t
$$

and

$$
E_{V}^{*}(\boldsymbol{a})=\int_{t^{0}}^{t^{1}} E_{V}(t, x, P(t, x), p) d t
$$


where all arguments are along $\boldsymbol{a}$ and $E_{V}$ is the function of (30-2). Then

$$
V(a)=V^{*}(a)+E_{V}^{*}(a)
$$

and

$$
\Delta V(a)=\Delta V^{*}(a)+E_{v}^{*}(a)
$$

Now by reasoning entirely analagous to that used in obtaining (43), then for any $\epsilon>0$ we can find a neighborhood $\mathscr{K}$ in $t x$ space about $\boldsymbol{a}_{0}$ such that for any admissible arc $\boldsymbol{a}$ in $\mathscr{K}$ we have

$$
\left|\Delta V^{*}(\boldsymbol{a})\right|<\epsilon\left[t^{1}-t^{0}\right]+\epsilon c^{-1}\left[t^{1}-t^{0}+E_{T}(\boldsymbol{a})\right] .
$$

Then by the $E_{T}$ domination of $V$ near $\boldsymbol{a}_{0}$, we have by reducing $\mathscr{K}$ if necessary that

$$
\begin{aligned}
|\Delta V(\boldsymbol{a})| & \leqq\left|\Delta V^{*}(\boldsymbol{a})\right|+\left|E_{V}^{*}(\boldsymbol{a})\right| \\
& <\epsilon\left[t^{1}-t^{0}\right]+\epsilon c^{-1}\left[t^{1}-t^{0}+E_{T}(\boldsymbol{a})\right]+c^{-1} E_{T}(\boldsymbol{a}) \\
& =\epsilon\left(1+c^{-1}\right)\left[t^{1}-t^{0}\right]+c^{-1}(1+\boldsymbol{\epsilon}) E_{T}(\boldsymbol{a}) .
\end{aligned}
$$

Now select $\epsilon$ such that

$$
\epsilon\left(1+c^{-1}\right)\left[t^{1}-t^{0}\right]<\epsilon^{\prime} \text { and } \epsilon c^{-1}<\epsilon^{\prime}
$$

Then the lemma is proven.

We next prove:

Lemma 4.4. Let $V$ satisfy the hypotheses of Lemma 4.3. Then given $\epsilon<0$, there exists $\eta>0$ and a neighborhood $\mathscr{L}$ of $a_{0}$ in txb space such that if $\boldsymbol{a}$ is admissible and in $\mathscr{L}$ and satisfies

$$
I_{0}(a) \leqq I_{0}\left(a_{0}\right)+\eta
$$

then

$$
\left|V(a)-V\left(a_{0}\right)\right|<\epsilon
$$

Proof. Assume the contrary, that is that there is a sequence $\left\{\boldsymbol{a}_{r}\right\}$ of admissible arcs which converge to $a_{0}$ uniformly in $t x b$ space such that

$$
I_{0}\left(a_{r}\right)-I_{0}\left(a_{0}\right) \leqq r^{-1} \text { and }\left|V\left(a_{r}\right)-V\left(a_{0}\right)\right|>\epsilon .
$$

By Lemma 4.2 given $\epsilon^{\prime}>0$, then if $r$ is large enough so that $\boldsymbol{a}_{r}$ is in the neighborhood $\mathscr{F}$ of that lemma, then 


$$
-\epsilon^{\prime}+\left(1-\epsilon^{\prime}\right) E_{T}\left(a_{r}\right)<I_{0}\left(a_{r}\right)-I_{0}\left(a_{0}\right) \leqq r^{-1} .
$$

Also, by Lemma 4.3, for $r$ large enough so that $a_{r}$ is in the neighborhood $\mathscr{K}$ of that lemma

(61) $\left|V\left(a_{r}\right)-V\left(a_{0}\right)\right|<\epsilon^{\prime}+\left(c^{-1}+\epsilon^{\prime}\right) E_{T}\left(a_{r}\right)<\epsilon^{\prime}+\left(c^{-1}+\epsilon^{\prime}\right) \frac{\left(r^{-1}+\epsilon^{\prime}\right)}{1-\epsilon^{\prime}}$.

Since this holds for all large $r$ and since $\epsilon^{\prime}$ can be made arbitrarily small, this gives a contradiction to the second part of (59) thus proving the Lemma.

With the help of these last three Lemmas, we can prove Theorem 4.1 as follows:

Proof. The integral of $K\left(a_{r}, a_{0}\right)$ defined in (25) involves the function $\bar{L}(t, x, p) \equiv L\left(p-p_{0}(t)\right)-1$. This function is $E_{T}$ dominated near $a_{0}$ on $R_{0}$ for the same reasons that (31) was true and furthermore satisfies

$$
\frac{\partial \bar{L}(t, x, p)}{\partial p^{i}}=0 \quad \text { along } \quad a_{0}
$$

so that $\bar{L}$ satisfies the hypothesis of Lemma 4.4 . Also, we note that

$$
\bar{L}\left(t, x, p_{0}(t)\right)=L\left(p_{0}(t)-p_{0}(t)\right)-1=0 .
$$

Then by Lemma 4.4 we have

$$
\lim _{r \rightarrow \infty} K\left(\boldsymbol{a}_{r}, \boldsymbol{a}_{0}\right)=0
$$

proving the first statement of Theorem 4.1.

Now by Hölder's Inequality

$$
\begin{aligned}
{\left[\int_{t^{0}}^{t^{1}}\left|p_{r}-p_{0}\right| d t\right]^{2} } & =\left[\int_{t^{0}}^{t^{1}}\left[L\left(p_{r}-p_{0}\right)-1\right]^{\frac{1}{2}}\left[L\left(p_{r}-p_{0}\right)+1\right]^{\frac{1}{2}} d t\right]^{2} \\
& \leqq K\left(\boldsymbol{a}_{r}, \boldsymbol{a}_{0}\right) \int_{t^{0}}^{t^{1}}\left[L\left(p_{r}-p_{0}\right)+1\right] d t \\
& =K\left(\boldsymbol{a}_{r}, \boldsymbol{a}_{0}\right)\left[2\left(t^{1}-t^{0}\right)+K\left(\boldsymbol{a}_{r}, \boldsymbol{a}_{0}\right)\right] .
\end{aligned}
$$

where $p_{r}, p_{0}$ mean $p$ evaluated respectively on $\boldsymbol{a}_{r}, \boldsymbol{a}_{0}$. Then by (64) we see that $p_{r}$ converges to $p_{0}$ [mean] which by standard theorems implies the existence of a subsequence $\left\{\boldsymbol{a}_{r_{\mathrm{r}}}\right\}$ satisfying the second statement of Theorem 4.1 and proving the theorem. 
5. Definition of the functions $\boldsymbol{\eta}_{r}, \boldsymbol{\alpha}_{r}, \boldsymbol{\beta}_{r}$. Now suppose that there is a sequence ${ }^{10}\left\{\boldsymbol{a}_{r}\right\}$ of admissible arcs converging to $\boldsymbol{a}_{0}$ uniformly in $t x b$ space with $I_{0}\left(\boldsymbol{a}_{r}\right) \leqq I_{0}\left(\boldsymbol{a}_{0}\right)$. Then by Theorem 4.1, we may replace this seqeunce by a subsequence which we again call $\left\{\boldsymbol{a}_{r}\right\}$ such that with control $p_{r}$ on $\boldsymbol{a}_{r}$,

$$
\left.\lim _{r} p_{r}=p_{0} \quad \text { [a. unif. }\right] \quad \text { on }\left[t^{0}, t^{1}\right]
$$

Also, the quantities

$$
b_{r}-b_{0}\left|, \quad \max _{t^{0} \leqq t \leqq t^{1}}\right| \Delta x_{r}(t) \mid
$$

$$
\int_{t^{0}}^{t^{1}} \dot{\mu}_{\alpha} \bar{\psi}^{\alpha}\left(t, x_{r}\right) d t, \quad \int_{t^{0}}^{t^{1}}\left|\dot{\mu}_{\alpha} \bar{\phi}^{\alpha}\left(t, x_{r}, p_{r}\right)\right| d t
$$

all converge in bounded manner to zero as $r \rightarrow \infty$ where: (i) $\bar{\psi}^{\alpha}, \bar{\phi}^{\alpha}$ are the functions introduced in (3), (ii) the subscript $r$, as usual denotes values on the arc $\boldsymbol{a}_{r}$, (iii) for the convergence of the integrals in (67) to zero we have used the fact that

$$
\int_{t^{0}}^{t^{1}} \dot{\mu}_{\alpha} \bar{\phi}^{\alpha}(t) d t=0 \quad \text { and } \quad \int_{t^{0}}^{t^{1}} \dot{\mu}_{\alpha} \bar{\psi}^{\alpha}(t) d t=0
$$

because of the properties of the functions $\dot{\mu}_{\alpha}(t)$.

Thus if we define:

$$
\begin{aligned}
k_{r}^{2}= & K\left(\boldsymbol{a}_{r}, \boldsymbol{a}_{0}\right)+\max \left[\int_{t^{0}}^{t^{1}} \dot{\mu}_{\alpha} \bar{\psi}^{\alpha} d t, \int_{t^{0}}^{t^{1}}\left|\dot{\mu}_{\alpha} \bar{\phi}^{\alpha}\right| d t\right] \\
& +\left|b_{r}-b_{0}\right|^{2}+\max _{\left[t^{0}, t^{1}\right]}\left|\Delta x_{r}(t)\right|^{2}
\end{aligned}
$$

where $\bar{\psi}^{\alpha}, \bar{\phi}^{\alpha}$ are evaluated along $\boldsymbol{a}_{r}$, then by Theorem 4.1 and the above statements, we have that

$$
\lim _{r \rightarrow \infty} k_{r}=0 \text {. }
$$

Now define the vector functions

10 Henceforth unless otherwise specified, all references to arcs $\boldsymbol{a}_{r}$ will mean members of subsequences (as defined in the following pages) of the sequence introduced here. 
(71-1) $\quad \eta_{r}^{i}(t) \equiv \Delta x_{r}^{i}(t) / k_{r} \quad \alpha_{r}^{k}(t) \equiv \Delta p_{r}^{k}(t) / k_{r} \quad \beta_{r}^{\sigma} \equiv \Delta b_{r}^{\sigma} / k_{r}$ and

$(71-2) h_{r}(t) \equiv 1+L\left(p_{r}(t)-p_{0}(t)\right)=k_{r}^{2}\left|\alpha_{r}(t)\right|^{2} /\left(L\left(p_{r}(t)-p_{0}(t)\right)-1\right)$

the second equality for $h_{r}$ following from the above definitions. Then by $(71-2)$

$$
\int_{t^{0}}^{t^{1}}\left|\alpha_{r}\right|^{2} / h_{r} d t=\int_{t^{0}}^{t^{1}}\left[L\left(p_{r}-p_{0}\right)-1\right] / k_{r}^{2} d t=K\left(a_{r}, a_{0}\right) / k_{r}^{2}
$$

which implies by the definition of $k_{r}$ that

$$
\beta_{r}^{2}+\max _{\left[t^{0}, t^{1}\right]}\left|\eta_{r}\right|^{2}+\int_{t^{0}}^{t^{1}} \frac{\left|\alpha_{r}\right|^{2}}{h_{r}} d t \leqq 1
$$

and hence in particular that the integral in (73) is uniformly bounded with respect to $r$.

We next prove:

LEMMA 5.1. The integrals of $h_{r}(t)$ are absolutely continuous uniformly with respect to $r$.

Proof. This follows because $h_{r}(t)$ differs by 2 from the integrand of $K\left(\boldsymbol{a}_{r}, \boldsymbol{a}_{0}\right)$ and $\lim _{r \rightarrow \infty} K\left(\boldsymbol{a}_{r}, \boldsymbol{a}_{0}\right)=0$.

LEMMA 5.2. The integrals $\int_{M} \alpha_{r} d t$ are absolutely continuous uniformly with respect to $r$.

Proof. By Hölder's Inequality and (73) we have that

$$
\left|\int_{M} \alpha_{r} d t\right|^{2} \leqq \int_{M}\left|\alpha_{r}\right|^{2} / h_{r} d t \cdot \int_{M} h_{r} d t \leqq \int_{M} h_{r} d t
$$

and by Lemma 5.1 this last integral is uniformly absolutely continuous.

6. Existence of the variation $\boldsymbol{\eta}_{0}$. We next establish a number of results concerning the convergence of $\eta_{r}, \alpha_{r}, \beta_{r}$. With $\left\{\boldsymbol{a}_{r}\right\}$ always as the sequence of arcs considered above we prove:

THEOREM 6.1. With $\beta_{r}$ defined in (71-1) then there exists a vector $\beta_{0}$ and a subsequence of arcs $\left\{\boldsymbol{a}_{r_{k}}\right\}$ which we again call $\left\{\boldsymbol{a}_{r}\right\}$ such that with $\left\{\beta_{r}\right\}$ as the associated values, then $\beta_{r}$ converges to $\beta_{0}$. 
Proof. This follows from the definitions of $k_{r}$ and $\beta_{r}$ and the Bolzano Weierstrass Theorem, applied to the original sequence $\left\{\beta_{r}\right\}$.

THEOREM 6.2. There exists a function $\alpha_{0}$ in $\mathscr{L}_{2}\left[t^{0}, t^{1}\right]$ and a subsequence of arcs $\left\{\boldsymbol{a}_{r_{k}}\right\}$ which we again call $\left\{\boldsymbol{a}_{r}\right\}$ such that with $\left\{\boldsymbol{\alpha}_{r}\right\}$ (defined in (71-1)) as the associated quantities, then for each bounded integrable function $g$

$$
\lim _{r \rightarrow \infty} \int_{t^{0}}^{t^{1}} g \alpha_{r} d t=\int_{t^{0}}^{t^{1}} g \alpha_{0} d t
$$

and if $s$ is a set on which $p_{r}$ converges uniformly to $p_{0}$ and $g$ is square integrable then

$$
\lim _{r \rightarrow \infty} \int_{s} g \alpha_{r} d t=\int_{s} g \alpha_{0} d t
$$

Proof. By the uniform boundedness of the integral in (73), there is a subsequence $\left\{\alpha_{r_{k}}\right\}$ which we again call $\left\{\alpha_{r}\right\}$ and a function $\bar{\alpha}_{0}$ in $\mathscr{L}_{2}\left[t^{0}, t^{1}\right]$ such that for every square integrable function $g$ and any measurable set $s$.

$$
\lim _{r \rightarrow \infty} \int \frac{g \alpha_{r} d t}{\sqrt{h_{r}}}=\int g \bar{\alpha}_{0} d t
$$

Now let $s$ be $\left[t^{0}, t^{1}\right]$ if $g$ is bounded and integrable while if $g$ is only square integrable, let $s$ be a set on which $p_{r}$ converges uniformly to $p_{0}$. Then we can write

$$
\int_{s} g \alpha_{r} d t=\sqrt{2} \int_{s} g \frac{\alpha_{r}}{\sqrt{h_{r}}} d t+\int_{s} g\left(\sqrt{h_{r}}-\sqrt{2}\right) \frac{\alpha_{r}}{\sqrt{h_{r}}} d t
$$

and by Hölder's Inequality together with (73)

$$
\begin{aligned}
{\left[\int_{s}\left|g\left(\sqrt{h_{r}}-\sqrt{2}\right) \frac{\alpha_{r}}{\sqrt{h_{r}}}\right| d t\right]^{2} } & \leqq \int_{s} g^{2}\left(\sqrt{h_{r}}-\sqrt{2}\right)^{2} d t \cdot \int_{s} \frac{\left|\alpha_{r}\right|^{2} d t}{h_{r}} \\
& \leqq \int_{s} g^{2}\left(\sqrt{h_{r}}-\sqrt{2}\right)^{2} d t
\end{aligned}
$$

Since $h_{r}(t) \geqq 2$, we have 
(80) $0 \leqq \int_{t^{0}}^{t^{1}}\left(\sqrt{h_{r}}-\sqrt{2}\right) d t \leqq \int_{t^{0}}^{t^{1}}\left(\sqrt{h_{r}}-\sqrt{2}\right)\left(\sqrt{h_{r}}+\sqrt{2}\right) d t$

$$
=\int_{t^{0}}^{t^{1}}\left(h_{r}-2\right) d t=\int_{t^{0}}^{t^{1}}\left[L\left(p_{r}-p_{0}\right)-1\right] d t=K\left(a_{r}, a_{0}\right) \rightarrow 0 \text { as } r \rightarrow \infty
$$

so that

$$
\lim _{r \rightarrow \infty} \int_{t^{0}}^{t^{1}}\left(\sqrt{h_{r}}-\sqrt{2}\right) d t=0
$$

Also since

$$
\int_{t^{0}}^{t^{1}}\left(\sqrt{h_{r}}-\sqrt{2}\right)^{2} d t=\int_{t^{0}}^{t^{1}}\left(h_{r}-2\right) d t-2 \sqrt{2} \cdot \int_{t^{0}}^{t^{1}}\left(\sqrt{h_{r}}-\sqrt{2}\right) d t
$$

then

$$
\lim _{r \rightarrow \infty} \int_{t^{0}}^{t^{1}}\left(\sqrt{h_{r}}-\sqrt{2}\right)^{2} d t=0
$$

Now let $g$ be a bounded integrable function and let $s$ be $\left[t^{0}, t^{1}\right]$ in the statements, (78) and (79). Then by (79), (83) and the boundedness of $g$ we see that

$$
\lim _{r \rightarrow \infty} \int_{s} g\left(\sqrt{h_{r}}-\sqrt{2}\right) \frac{\alpha_{r}}{\sqrt{h_{r}}} d t=0
$$

so that by using (77), (78) and defining $\alpha_{0}=\sqrt{2} \bar{\alpha}_{0}$ we have

(85) $\lim _{r \rightarrow \infty} \int_{s} g \alpha_{r} d t=\lim _{r \rightarrow \infty} \sqrt{2} \int_{s} g \frac{\alpha_{r}}{\sqrt{h_{r}}} d t=\sqrt{2} \int_{s} g \bar{\alpha}_{0} d t=\int_{s} g \alpha_{0} d t$

proving the first statement of the Theorem.

In order to prove the second statement let $g$ be in $\mathscr{L}_{2}\left[t^{0}, t^{1}\right]$ and let $s$ be a set on which $p_{r}$ converges to $p_{0}$ uniformly. Then our statements (78) and (79) hold also in this case, and on $s$ we have

$$
\lim _{r \rightarrow \infty}\left(h_{r}-2\right)=0 \quad \text { uniformly on } s
$$

so that (84) and (85) hold also in this case, proving the Theorem. 
Now with $\left\{\boldsymbol{a}_{r}\right\}$ as the sequence of arcs yielded by the previous two theorems and with $\eta_{r}(t)$ defined in (71-1) as the associated quantities, we next prove:

THEOREM 6.3. There exists a function $\eta_{0}(t)$ on $\left[t^{0}, t^{1}\right]$ with derivative $\dot{\eta}_{0}(t)$ in $\mathscr{L}_{2}\left[t^{0}, t^{1}\right]$ and a subsequence of arcs $\left\{a_{r_{k}}\right\}$ which we again call $\left\{a_{r}\right\}$ such that $\eta_{r}(t)$ converges to $\eta_{0}(t)$ uniformly on $\left[t^{0}, t^{1}\right]$. Furthermore, if $g$ is a bounded integrable function on $\left[t^{0}, t^{1}\right]$, we have

$$
\lim _{r \rightarrow \infty} \int_{t^{0}}^{t^{1}} g \dot{\eta}_{r} d t=\int_{t^{0}}^{t^{1}} g \dot{\eta}_{0} d t
$$

while if $g$ is in $\mathscr{L}_{2}\left[t^{0}, t^{1}\right]$ and if $s$ is a set on which $p_{r}$ converges to $p_{0}$ uniformly, then

$$
\lim _{r \rightarrow \infty} \int_{s} g \dot{\eta}_{r} d t=\int_{s} g \dot{\eta}_{0} d t
$$

where $\dot{\eta}_{r}$ is the derivative of $\eta_{r}$.

Proof. We note that

$$
\dot{\eta}_{r}(t)=\left[\dot{x}_{r}(t)-\dot{x}_{0}(t)\right] / k_{r}=\left[f\left(t, x_{r}(t), p_{r}(t)\right)-f(t)\right] / k_{r} .
$$

Now by assumption (vi), we have

$$
\left|\dot{\eta}_{r}(t)\right|^{2} \leqq \rho^{2}\left[\left|\eta_{r}(t)\right|^{2}+\left|\alpha_{r}(t)\right|^{2}\right]
$$

so that

$$
\left|\eta_{r}(t)\right|^{2}>\rho^{-2}\left|\dot{\eta}_{r}(t)\right|^{2}-\left|\alpha_{r}(t)\right|^{2}
$$

Then by dividing (90) by $h_{r}$ and integrating and then adding that inequality to (73) we get

$$
\beta_{r}^{2}+\max _{\left[t^{0}, t^{1}\right]}\left|\eta_{r}\right|^{2}+\frac{1}{\rho^{2}} \int_{t^{0}}^{t^{1}}\left|\dot{\eta}_{r}\right|^{2} / h_{r} d t<1+\int_{t^{0}}^{t^{1}}\left|\eta_{r}\right|^{2} / h_{r} d t .
$$

Also by the definitions of $k_{r}$ and $h_{r}$ we see that

$$
\max _{\left[t^{0}, t^{1}\right]}\left|\eta_{r}\right|^{2} / h_{r} \leqq \frac{1}{2}
$$

so that 


$$
\int_{t^{0}}^{t^{1}}\left|\eta_{r}\right|^{2} / h_{r} d t \leqq \frac{1}{2}\left[t^{1}-t^{0}\right]
$$

and then by (91), the first integral there is uniformly bounded with respect to $r$, that is

$$
\int_{t^{0}}^{t^{1}}\left|\dot{\eta}_{r}\right|^{2} / h_{r} d t \leqq \Gamma \quad \text { uniformly with respect to } r \text {. }
$$

Then by using an argument similar to that used in the previous Theorem, there exists a function $\dot{\eta}_{0}(t)$ on $\mathscr{L}_{2}\left[t^{0}, t^{1}\right]$ such that the conclusions of the present theorem which concern the function $\dot{\eta}_{0}$ are true.

In order now to prove the remaining item of our Theorem, set

$$
\eta_{0}^{\prime}(t)=X_{b^{\alpha}}^{t^{\alpha}} \beta_{0}^{\sigma}+\int_{t^{0}}^{t^{1}} \dot{\eta}_{0}^{\prime} d t \quad t^{0} \leqq t \leqq t^{1} \quad i=1, \cdots, N
$$

where $X^{i 0}$ are the functions of $(1-4), \beta_{0}$ is the vector of Theorem 6.1, and $X_{b^{\circ}}^{\prime 0}$ are evaluated on $\boldsymbol{a}_{0}$.

By Hölder's Inequality together with (94) we have for any measurable set $M$

$$
\begin{aligned}
{\left[\int_{M}\left|\dot{\eta}_{r}\right| d t\right]^{2} } & \leqq \int_{M}\left|\dot{\eta}_{r}\right|^{2} / h_{r} d t \cdot \int_{M} h_{r} d t \\
& \leqq \int_{t^{0}}^{t^{1}}\left|\dot{\eta}_{r}\right|^{2} / h_{r} d t \cdot \int_{M} h_{r} d t \leqq \Gamma \int_{M} h_{r} d t
\end{aligned}
$$

Then by the uniform absolute continuity of the integrals of. $h_{r}$ (Lemma 5.1) we see that the functions

$$
\eta_{r}^{\prime}(t)=k_{r}^{-1} \Delta x_{r}^{\prime}\left(t^{0}\right)+\int_{t^{0}}^{t} \dot{\eta}_{r}^{\prime} d t \quad t^{0} \leqq t \leqq t^{1}
$$

are absolutely continuous uniformly with respect to $r$.

Also by (86) which we've already proven,

$$
\lim _{r \rightarrow \infty} \int_{t^{10}}^{t} \dot{\eta}_{r} d t=\int_{t^{0}}^{t} \dot{\eta}_{0} d t \quad t^{0} \leqq t \leqq t^{1}
$$

Furthermore by Theorem 4.3 and the admissibility of our arcs we have that

$$
\lim _{r \rightarrow \infty} \Delta x_{r}^{t}\left(t^{0}\right) / k_{r}=\lim _{r \rightarrow \infty} \frac{\Delta X^{i 0}\left(b_{r}\right)}{\Delta b_{r}^{\sigma}} \beta_{r}^{\sigma}=X_{b^{\circ}}^{i 0} \beta_{0}^{\sigma} .
$$


Thus by (97) through (99), the definition (95) and the uniform absolute continuity of $\eta_{r}^{\prime}(t)$ we see that

$$
\lim _{r \rightarrow \infty} \eta_{r}^{i}(t)=\eta_{0}^{i}(t) \quad \text { uniformly on }\left[t^{0}, t^{1}\right]
$$

thus proving the theorem.

We have thus proven that if we define $\eta_{0}$ as the variation

$$
\eta_{0}: \eta_{0}(t) \quad \alpha_{0}(t) \quad \beta_{0} \quad t^{0} \leqq t \leqq t^{1}
$$

and $\eta_{r}$ as the vector functions

$$
\eta_{r}: \eta_{r}(t) \quad \alpha_{r}(t) \quad \beta_{r} \quad t^{0} \leqq t \leqq t^{1}
$$

then $\eta_{r}$ converges to $\eta_{0}$ in the sense that

$$
\begin{aligned}
& \beta_{r} \text { converges to } \beta_{0} \\
& \left.\begin{array}{l}
\eta_{r}(t) \text { converges uniformly to } \eta_{0}(t) \\
\alpha_{r}(t) \text { converges [a. unif.] to } \alpha_{0}(t)
\end{array}\right\} t^{0} \leqq t \leqq t^{1} \text {. }
\end{aligned}
$$

7. Additional properties of the variation $\boldsymbol{\eta}_{0}$. Now, using the functions $P(t, x)$ of Lemma 4.1, we recall that by our convention $P^{k}(t) \equiv P^{k}\left(t, x_{0}(t)\right)=p_{0}^{k}(t)$ the value of the control on $a_{0}$. For convenience, let us denote this as $P_{0}^{k}(t)$. Thus $P_{0}^{k}(t)=p_{0}^{k}(t)$. Next denote by $P_{r}(t)$ the values of $P$ along the arc $\left(a_{r}\right)$, i.e.,

$$
P_{r}^{k}(t) \equiv P^{k}\left(t, x_{r}(t)\right) \quad k=1, \cdots, K \quad t^{0} \leqq t \leqq t^{1}
$$

and also define the vector functions

$$
\rho_{r}(t) \equiv \frac{P_{r}(t)-P_{0}(t)}{k_{r}} \quad \rho_{0}(t) \equiv P_{x^{\prime}}\left(t, x_{0}(t)\right) \eta_{0}^{j}(t)
$$

Then we state the following results which are proven in an analagous manner to Lemmas 8.1 through 8.4 of [1] except that derivatives of state functions are replaced by controls.

LEMMA 7.1. The following relations are true:

$$
\phi^{\alpha}\left(t, x_{r}, P_{r}\right)=\phi^{\alpha}\left(t, x_{0}, P_{0}\right) \quad \alpha=1, \cdots, m
$$

(where $\phi^{\alpha}$ are the functions introduced above (2)) 
(105-2) $\quad \lim _{r \rightarrow \infty} P_{r}(t)=P_{0}(t)=p_{0}(t)$ uniformly on $\left[t^{0}, t^{1}\right]$

(105-3) $\quad \lim _{r \rightarrow \infty} \rho_{r}(t)=\rho_{0}(t)$ uniformly on $\left[t^{0}, t^{1}\right]$.

If $N_{r}^{k}(t)$ are continuous functions which converge uniformly to $N_{0}^{k}(t)$ on $\left[t^{0}, t^{1}\right]$ and if $g$ is square integrable then

$$
\lim _{r \rightarrow \infty} \int_{s} N_{r}^{k}\left(\alpha_{r}^{k}-\rho_{r}^{k}\right) d t=\int_{s} N_{0}^{k}\left(\alpha_{0}^{k}-\rho_{0}^{k}\right) d t
$$

for every measurable set $s$ in $\left[t^{0}, t^{1}\right]$ and

$$
\lim _{r \rightarrow \infty} \int_{s} g\left(\alpha_{r}^{k}-\rho_{r}^{k}\right) d t=\int_{s} g\left(\alpha_{0}^{k}-\rho_{0}^{k}\right) d t
$$

for every measurable set $s$ in $\left[t^{0}, t^{1}\right]$ upon which $p_{r}(t)$ converges uniformly to $p_{0}(t)$. Furthermore if $\mathscr{W}(t, x, p)$ is any function of class $C^{\prime}$ near $\boldsymbol{a}_{0}$, then

$$
\lim _{r \rightarrow \infty} k_{r}^{-1}\left[\mathcal{W}\left(t, x_{r}, P_{r}\right)-\mathscr{W}\left(t, x_{0}, P_{0}\right)\right]=\mathscr{W}_{x^{\prime}} \eta_{0}^{i}+\mathscr{W}_{p^{k}} \rho_{0}^{k}
$$

uniformly on $\left[t^{0}, t^{1}\right]$.

Finally with the functions $\phi^{\alpha}$ of $(105-1)$ we have

$$
\phi_{x^{i}}^{\alpha_{i}} \eta_{0}^{t}+\phi_{p^{k}}^{\alpha_{k}} \rho_{0}^{k}=0 \quad \alpha=1, \cdots, m \quad t^{0} \leqq t \leqq t^{1} .
$$

Now using the functions $P(t, x)$ of Lemma 4.1 we extend to any measurable set $s$ in $\left[t^{0}, t^{1}\right]$, the technique used to break up functionals in (50) as follows:

Let $\mathscr{V}(t, x, p)$ be a function of class $C^{\prime}$ near $\boldsymbol{a}_{0}$ and $\boldsymbol{a}$ an arc and define

$$
\mathscr{V}(a, s)=\int_{s} \mathscr{V}(t, x, p) d t
$$

$$
\mathscr{V}^{*}(a, s)=\int_{s}\left(\mathscr{V}(t, x, P)+\left(p^{k}-P^{k}\right) \mathscr{V}_{p^{k}}(t, x, P)\right] d t
$$

$$
E_{v}^{*}(\boldsymbol{a}, s)=\int_{s} E_{\mathcal{r}}(t, x, P, p) d t
$$

Also, for a variation $\eta$ of the arc $a_{0}$ 


$$
\eta: \eta(t) \quad \alpha(t) \quad \beta \quad t^{0} \leqq t \leqq t^{1}
$$

define

$$
\mathscr{V}_{1}(\eta, s)=\int_{s}\left[\mathscr{V}_{x^{i}} \eta^{i}+\mathscr{V}_{p^{k}} \alpha^{k}\right] d t
$$

where: (i) all values of $x, p, P$ in (109-1) through (109-3) are along the arc $a$, (ii) the function $E_{V}$ in (109-3) is the Weierstrass $E$ function for $\mathscr{V}$, and (iii) the arguments of $\mathscr{V}_{x^{i}}, \mathscr{V}_{p^{k}}$ in (109-4) are from $a_{0}$. Then as in (52) we see that

$$
\mathscr{V}(\boldsymbol{a}, s)=\mathscr{V}^{*}(\boldsymbol{a}, s)+E_{V}^{*}(\boldsymbol{a}, s)
$$

and, in analagous manner to Lemma 9.1 of [1] we have

LEMMA 7.2. If $\mathscr{V}(t, x, p)$ is of class $C^{\prime}$ near $a_{0}$, then

$$
\lim _{r \rightarrow \infty} k_{r}^{-1}\left[\mathscr{V}^{*}\left(\boldsymbol{a}_{r}, s\right)-\mathscr{V}^{*}\left(\boldsymbol{a}_{0}, s\right)\right]=\mathscr{V}_{1}\left(\eta_{0}, s\right)
$$

8. Evaluation of second order terms. Our ultimate purpose now is to prove the admissibility of the variation $\eta_{0}$ defined in (101) and constructed in Theorems 4.3 through 5.1. As a first step in this procedure, we evaluate certain second order terms.

Continuing with our sequence $\left\{\boldsymbol{a}_{r}\right\}$, then dividing (36) by $k_{r}^{2}$, evaluating the expression on this sequence, moving most of the terms to the left side and taking superior limits, we obtain

(112) $\limsup _{r \rightarrow \infty} k_{r}^{-2}\left[-\Delta J_{T}^{*}\left(\boldsymbol{a}_{r}\right)-\mu_{\alpha}\left(t^{0}\right) \Delta \psi^{\alpha}\left(t^{0}, x_{r}\left(t^{0}\right)\right)\right]$

$$
\begin{aligned}
& +\limsup _{r \rightarrow \infty} k_{r}^{-2}\left[-B_{T}^{*}\left(\boldsymbol{a}_{r}\right)\right]+\limsup _{r \rightarrow \infty} k_{r}^{-2} \lambda_{\gamma} \Delta I_{\gamma}\left(\boldsymbol{a}_{r}\right) \\
& +\limsup _{r \rightarrow \infty} k_{r}^{-2} \mu_{\alpha}\left(t^{1}\right) \Delta \psi^{\alpha}\left(t^{1}, x_{r}\left(t^{1}\right) \geqq \limsup _{r \rightarrow \infty} k_{r}^{-2} E_{T}\left(\boldsymbol{a}_{r}\right) .\right.
\end{aligned}
$$

In order to establish the admissibility of $\eta_{0}$, we shall have to deal with the separate terms of (112). The first term to come under consideration is the integral part of $\Delta J_{T}^{*}\left(\boldsymbol{a}_{r}\right)$ which we denote by the symbol $\Delta_{I} J_{T}^{*}\left(\boldsymbol{a}_{r}\right)$. Thus

$(113-1) \quad \Delta_{I} J_{T}^{*}\left(a_{r}\right) \equiv \int_{t^{0}}^{t^{1}}\left[G\left(t, x_{r}, P_{r}\right)-G(t)+\left(p_{r}^{k}-P_{r}^{k}\right) G_{p^{k}}\left(t, x_{r}, P_{r}\right)\right] d t$. 
With $\eta_{0}(t), \alpha_{0}(t)$ as quantities associated with the variation $\eta_{0}$ and $\rho_{r}$ as the terms of (104) and finally with $\omega_{G}\left(\eta_{0}, \alpha_{0}\right)$ denoting the quadratic form

$$
(113-2) \omega_{G}\left(\eta_{0}, \alpha_{0}\right) \equiv G_{x^{\prime} x^{\prime}} \eta_{0}^{i} \eta_{0}^{j}+2 G_{x^{i} p^{k}} \eta_{0}^{i} \alpha_{0}^{k}+G_{p^{h} p^{k} \alpha_{0}^{h} \alpha_{0}^{k}} \quad \begin{array}{r}
i, j=1, \cdots, N \\
h, k=1, \cdots, K
\end{array}
$$

where the partial derivatives are formed along $\boldsymbol{a}_{0}$, we next prove:

LEMMA 8.1 .

$$
\begin{gathered}
(114) \lim _{r \rightarrow \infty} k_{r}^{-2} \Delta_{I} J_{T}^{*}\left(\boldsymbol{a}_{r}\right)=\frac{1}{2} \int_{t^{0}}^{t^{1}}\left[\omega_{G}\left(\eta_{0}, \alpha_{0}\right)-G_{p^{h} p^{k}}\left(\alpha_{0}^{h}-\rho_{0}^{h}\right)\left(\alpha_{0}^{k}-\rho_{0}^{k}\right)\right] d t \\
i, j=1, \cdots, N \quad h, k=1, \cdots, K .
\end{gathered}
$$

Proof. By Taylor's Theorem and the definitions of $P_{r}, \rho_{r}$

$$
\begin{aligned}
& \frac{G\left(t, x_{r}, P_{r}\right)-G(t)+\left(p_{r}^{k}-P_{r}^{k}\right) G_{p^{k}}\left(t, x_{r}, P_{r}\right)}{k_{r}^{2}} \\
& =\frac{k_{r}\left[G_{x^{\prime}} \eta_{r}^{i}+G_{p^{k}} \rho_{r}^{k}+\left(\alpha_{r}^{k}-\rho_{r}^{k}\right) G_{p^{k}}\right]}{k_{r}^{2}} \\
& +\frac{k_{r}^{2}\left(\alpha_{r}^{k}-\rho_{r}^{k}\right)\left[\tilde{\tilde{G}}_{p^{k} p^{h}} \rho_{r}^{h}+\tilde{\tilde{G}}_{p^{k} x^{k}} \eta_{r}^{i}\right]}{k_{r}^{2}} \\
& +\frac{\frac{k_{r}^{2}}{2}\left[\tilde{G}_{x^{i} x^{i}} \eta_{r}^{i} \eta_{r}^{j}+2 \tilde{G}_{x^{i} p^{k}} \eta_{r}^{i} \rho_{r}^{k}+\tilde{G}_{p^{h} p^{k}} \rho_{r}^{h} \rho_{r}^{k}\right]}{k_{r}^{2}}
\end{aligned}
$$

where: (i) $G_{x^{i}}, G_{p^{h}}$ are evaluated on the arc $a_{0}$ and (ii) $\tilde{G}_{x^{i} x^{i}}, \tilde{G}_{x^{i} p^{k}}, \tilde{G}_{p^{h} p^{h}}$, $\tilde{\tilde{G}}_{p^{k} p^{h}}, \tilde{\tilde{G}}_{p^{h} x^{\prime}}$ are all evaluated at intermediate points on the line segment

$$
\left(t, x_{0}+\theta \Delta x_{r}, P_{0}+\theta \Delta P_{r}\right) \quad 0<\theta<1 \text {. }
$$

Now using (10) together with Lemma 7.1 and the uniform convergence of $x_{r}$ to $x_{0}$ and $\eta_{r}$ to $\eta_{0}$ on $\left[t^{0}, t^{1}\right]$ we see by integrating (115) that

(116) $\lim _{r \rightarrow \infty} k_{r}^{-2} \Delta_{I} J_{T}^{*}\left(a_{r}\right)=\frac{1}{2} \int_{t^{0}}^{t^{1}}\left[G_{x^{i} x^{i}} \eta_{0}^{i} \eta_{0}^{j}+2 G_{x^{i} p^{k}} \eta_{0}^{i} \rho_{0}^{k}+G_{p^{h} p^{k}} \rho_{0}^{h} \rho_{0}^{k}\right.$

$$
\left.+2\left(\alpha_{0}^{k}-\rho_{0}^{k}\right)\left(G_{p^{h} p^{k}} \rho_{0}^{h}+G_{p^{k} x^{i}} \eta_{0}^{j}\right)\right] d t
$$

where all partial derivatives are evaluated along $\boldsymbol{a}_{0}$. Combining terms this becomes 
(117) $\lim _{r \rightarrow \infty} k_{r}^{-2} \Delta_{I} J_{T}^{*}\left(a_{r}\right)=\frac{1}{2} \int_{t^{0}}^{t^{1}}\left[G_{x^{i} x^{i}} \eta_{0}^{i} \eta_{0}^{j}+2 G_{x^{\prime} p^{k}} \eta_{0}^{i} \alpha_{0}^{k}-G_{p^{h} p^{k}} \rho_{0}^{h} \rho_{0}^{k}\right.$

$$
\left.+2 G_{p^{h} p^{k}} \rho_{0}^{h} \alpha_{0}^{k}\right] d t .
$$

Now adding and subtracting the terms $G_{p^{h} p^{k}} \alpha_{0}^{h} \alpha_{0}^{k}$ in the above integrand produces (114) and the lemma is proven.

Next, by using the definition of $\Delta J_{T}^{*}\left(\boldsymbol{a}_{r}\right)$ together with Lemma 8.1, the transversality relation (8) and the relation for $\lambda_{p+i}$ in (4-1) we get

(118) $\limsup _{r \rightarrow \infty} k_{r}^{-2}\left[-\Delta J_{T}^{*}\left(\boldsymbol{a}_{r}\right)-\mu_{\alpha}\left(t^{0}\right) \Delta \psi^{\alpha}\left(t^{0}, x_{r}\left(t^{0}\right)\right)\right]$

$$
\begin{gathered}
=\limsup _{r \rightarrow \infty} k_{r}^{-2}\left[K^{\alpha} \psi_{x^{(}}^{\alpha}\left(t^{0}\right) X_{b^{\alpha}}^{\prime 0} \Delta b_{r}^{\sigma}-\mu_{\alpha}\left(t^{0}\right) \Delta \psi^{\alpha}\left(t^{0}, x_{r}\left(t^{0}\right)\right)\right] \\
-\frac{1}{2} \int_{t^{0}}^{t^{1}}\left[\omega_{G}\left(\eta_{0}, \alpha_{0}\right)-G_{p^{h} p^{k}}\left(\alpha_{0}^{h}-\rho_{0}^{h}\right)\left(\alpha_{0}^{k}-\rho_{0}^{k}\right)\right] d t .
\end{gathered}
$$

As a next step we prove

LEMMA 8.2.

(119)

$$
\begin{aligned}
\limsup _{r \rightarrow \infty} & k_{r}^{-2}\left[K^{\alpha} \psi_{x^{\prime}}^{\alpha}\left(t^{0}\right) X_{b^{\alpha}}^{i^{0}} \Delta b_{r}^{\sigma}-\mu_{\alpha}\left(t^{0}\right) \Delta \psi^{\alpha}\left(t^{0}, x_{r}\left(t^{0}\right)\right)\right] \\
\leqq & \limsup _{r \rightarrow \infty} k_{r}^{-2}\left[-\left(\mu_{\alpha}\left(t^{0}\right)-K^{\alpha}\right) \psi_{x^{\prime}}^{\alpha}\left(t^{0}\right) \Delta x_{r}^{i}\left(t^{0}\right)\right] \\
& -\frac{1}{2}\left[K^{\alpha} \psi_{x^{\prime}}^{\alpha}\left(t^{0}\right) X_{b^{\sigma} b^{r}}^{i 0}+K^{\alpha} \psi_{x^{i} x^{\prime}}^{\alpha}\left(t^{0}\right) X_{b^{\sigma}}^{i 0} X_{b^{\top}}^{i 0}\right] \beta_{0}^{\sigma} \beta_{0}^{\tau} \\
& -\frac{1}{2}\left(\mu_{\alpha}\left(t^{0}\right)-K^{\alpha}\right) \psi_{x^{\prime} x^{\prime}}^{\alpha}\left(t^{0}\right) \eta_{0}^{i}\left(t^{0}\right) \eta_{0}^{j}\left(t^{0}\right) .
\end{aligned}
$$

Proof. By Taylor's Theorem and the admissibility of our arcs, we have

$$
X_{b^{\circ}}^{i 0} \Delta b_{r}^{\sigma}=\Delta x_{r}^{i}\left(t^{0}\right)-\frac{1}{2} \tilde{X}_{b^{\sigma} b^{\top}}^{i 0} \Delta b_{r}^{\sigma} \Delta b_{r}^{\tau}
$$

and

$(120-2) \quad \Delta \psi^{\alpha}\left(t^{0}, x_{r}\left(t^{0}\right)\right)=\psi_{x^{i}}^{\alpha}\left(t^{0}\right) \Delta x_{r}^{i}\left(t^{0}\right)+\frac{1}{2} \tilde{\psi}_{x^{i} x^{\prime}}^{\alpha} \Delta x_{r}^{i}\left(t^{0}\right) \Delta x_{r}^{\prime}\left(t^{0}\right)$

where $\tilde{X}_{b^{b} b^{\tau}}^{i 0}, \tilde{\psi}_{x^{\prime} x^{i}}^{\alpha}$ are intermediate values on the line segments 


$$
b_{0}+\theta \Delta b_{r} \quad \text { and } t^{0}, x_{0}\left(t^{0}\right)+\theta \Delta x_{r}\left(t^{0}\right) \quad 0<\theta<1 .
$$

Then by using (120) and combining terms, we have that

$$
K^{\alpha} \psi_{x}^{\alpha}\left(t^{0}\right) X_{b}^{i 0} \Delta b_{r}^{\sigma}-\mu_{\alpha}\left(t^{0}\right) \Delta \psi^{\alpha}\left(t^{0}, x_{r}\left(t^{0}\right)\right)=-\left(\mu_{\alpha}\left(t^{0}\right)-K^{\alpha}\right) \psi_{x}^{\alpha}\left(t^{0}\right) \Delta x_{r}^{i}\left(t^{0}\right)
$$

$$
-\frac{1}{2} K^{\alpha} \psi_{x^{i}}^{\alpha}\left(t^{0}\right) \tilde{X}_{b^{\sigma} b^{\tau}}^{i 0} \Delta b_{r}^{\sigma} \Delta b_{r}^{\tau}-\frac{1}{2} \mu_{\alpha}\left(t^{0}\right) \tilde{\psi}_{x^{i} x^{i}}^{\alpha} \Delta x_{r}^{i}\left(t^{0}\right) \Delta x_{r}^{j}\left(t^{0}\right) .
$$

Now by the admissibility of our arcs, the terms,

$$
\Delta x_{r}^{i}\left(t^{0}\right) \quad \Delta X^{i 0}\left(b_{r}\right)
$$

(where $\Delta X^{i 0}\left(b_{r}\right)$ means $X^{i 0}\left(b_{r}\right)-X^{i 0}\left(b_{0}\right)$ ) are equal, so that we may add to and subtract from (121) the respective quantities

$$
\frac{1}{2} K^{\alpha} \tilde{\psi}_{x^{\prime} x^{\prime}}^{\alpha} \Delta x_{r}^{\prime}\left(t^{0}\right) \Delta x_{r}^{j}\left(t^{0}\right) \text { and } \frac{1}{2} K^{\alpha} \tilde{\psi}_{x^{i} x}^{\alpha} \Delta X^{i 0}\left(b_{r}\right) \Delta X^{\prime 0}\left(b_{r}\right)
$$

(where $\tilde{\psi}_{x^{\prime} x^{\prime}}^{\alpha}$ here means evaluation at the same point as for that term in (121)) to get

$$
\begin{aligned}
& K^{\alpha} \psi_{x^{\prime}}^{\alpha}\left(t^{0}\right) X_{b^{\alpha}}^{i 0} \Delta b_{r}^{\sigma}-\mu_{\alpha}\left(t^{0}\right) \Delta \psi^{\alpha}\left(t^{0}, x_{r}\left(t^{0}\right)\right)=-\left(\mu_{\alpha}\left(t^{0}\right)-K^{\alpha}\right) \psi_{x^{\prime}}^{\alpha}\left(t^{0}\right) \Delta x_{r}^{i}\left(t^{0}\right) \\
& -\frac{1}{2} K^{\alpha} \psi_{x^{\prime}}^{\alpha}\left(t^{0}\right) \tilde{X}_{b^{\alpha} b^{\tau}}^{i 0} \Delta b_{r}^{\sigma} \Delta b_{r}^{\tau}-\frac{1}{2} K^{\alpha} \tilde{\psi}_{x^{i} x^{i}}^{\alpha} \Delta X^{i 0}\left(b_{r}\right) \Delta X^{j 0}\left(b_{r}\right) \\
& -\frac{1}{2}\left(\mu_{\alpha}\left(t^{0}\right)-K^{\alpha}\right) \tilde{\psi}_{x^{i} x^{j}}^{\alpha} \Delta x_{r}^{i}\left(t^{0}\right) \Delta x_{r}^{j}\left(t^{0}\right) .
\end{aligned}
$$

Now, dividing by $k_{r}^{2}$, taking superior limits and using the definition of $\beta_{0}$ yields (119) proving the lemma.

The next term of (112) which we consider is the one involving $B_{T}^{*}\left(a_{r}\right)$. We prove:

\section{LEMMA 8.3 .}

$$
\lim _{r \rightarrow \infty} k_{r}^{-2} B_{T}^{*}\left(a_{r}\right)=\frac{1}{2}\left[\left(z_{l}\left(t^{s}\right) X_{b}^{i s \sigma_{b} \tau}\right)_{s=0}^{s=1}+g_{0_{b} \sigma_{b} \tau}+\lambda_{\gamma} g_{\gamma_{b} \sigma_{b} \tau}\right] \beta_{0}^{\sigma} \beta_{0}^{\tau}
$$

Proof. By Taylor's Theorem and (27-3) we have

(126) $B_{r}^{*}\left(\boldsymbol{a}_{r}\right)=\frac{1}{2}\left[\left(z^{l}\left(t^{s}\right) \tilde{X}_{b^{\prime} b^{\tau}}^{s s}\right)_{s=0}^{s=1}+\tilde{g}_{0_{b^{\prime} \sigma^{\sigma^{*}}}}+\lambda_{\gamma} \tilde{g}_{\gamma_{b} \sigma_{b}}\right] \Delta b_{r}^{\sigma} \Delta b_{r}^{\tau} \quad \sigma, \tau=1, \cdots, K$ 
where $\tilde{X}_{b}^{i s} \sigma_{b} r, \tilde{g}_{0_{b} \sigma_{b} \tau}, \tilde{g}_{\gamma_{b} \sigma_{b} \tau}$ indicate intermediate values on the line segment $b_{0}+\theta \Delta b_{r} \quad 0<\theta<1$. By dividing by $k_{r}^{2}$, taking limits and using the definition of $B_{0}$ in (101) we obtain (125).

Now by using (118) and (112) together with Lemmas 8.2 and 8.3 we obtain

$$
\begin{aligned}
& -\frac{1}{2}\left[\left(z^{i}\left(t^{s}\right) X_{b}^{i s} b_{b}\right)_{s=0}^{s=1}+g_{0_{b} \sigma_{b} \tau}+\lambda_{\gamma} g_{\gamma_{b} b_{b} \tau}+K^{\alpha} \psi_{x^{\prime}}^{\alpha}\left(t^{0}\right) X_{b^{\sigma} b^{\tau}}^{i 0}\right. \\
& \left.+K^{\alpha} \psi_{x^{\prime} x^{\prime}}^{\alpha}\left(t^{0}\right) X_{b^{\sigma}}^{i 0} X_{b^{\top}}^{j^{0}}\right] \beta_{0}^{\sigma} \beta_{0}^{\tau} \\
& -\frac{1}{2} \int_{t^{0}}^{t^{1}}\left[\omega_{G}\left(\eta_{0}, \alpha_{0}\right)-G_{p^{h} p^{k}}\left(\alpha_{0}^{h}-\rho_{0}^{h}\right)\left(\alpha_{0}^{k}-\rho_{0}^{k}\right)\right] d t \\
& -\frac{1}{2}\left(\mu_{\alpha}\left(t^{0}\right)-K^{\alpha}\right) \psi_{x^{i} x^{\prime}}^{\alpha_{1}}\left(t^{0}\right) \eta_{0}^{\prime}\left(t^{0}\right) \eta_{0}^{\prime}\left(t^{0}\right) \\
& +\limsup _{r \rightarrow \infty} k_{r}^{-2}\left[-\left(\mu_{\alpha}\left(t^{0}\right)-K^{\alpha}\right) \psi_{x}^{\alpha}\left(t^{0}\right) \Delta x_{r}^{i}\left(t^{0}\right)\right] \\
& +\limsup k_{r}^{-2} \lambda_{\gamma} \Delta I_{\gamma}\left(\boldsymbol{a}_{r}\right)+\limsup k_{r}^{-2} \mu_{\alpha}\left(t^{1}\right) \Delta \psi^{\alpha}\left(t^{1}, x_{r}\left(t^{1}\right)\right) \\
& \geqq \lim \sup k_{r}^{-2} E_{T}\left(\boldsymbol{a}_{r}\right) \text {. }
\end{aligned}
$$

Referring to (24) and (5) for the definition of $J_{2}\left(a_{0}, \eta_{0}\right)$ and $\mathscr{G}$ we see that (127) gives

$$
\begin{aligned}
& -\frac{1}{2} J_{2}\left(a_{0}, \eta_{0}\right)+\frac{1}{2} \int_{t^{0}}^{t^{1}} G_{p^{h} p^{k}}\left(\alpha_{0}^{h}-\rho_{0}^{h}\right)\left(\alpha_{0}^{k}-\rho_{0}^{k}\right) d t \\
& -\frac{1}{2}\left(\mu_{\alpha}\left(t^{0}\right)-K^{\alpha}\right) \psi_{x^{2} x^{\prime}}^{\alpha}\left(t^{0}\right) \eta_{0}^{i}\left(t^{0}\right) \eta_{0}^{j}\left(t^{0}\right) \\
& +\limsup _{r \rightarrow \infty} k_{r}^{-2}\left[-\left(\mu_{\alpha}\left(t^{0}\right)-K^{\alpha}\right) \psi_{x^{*}}^{\alpha}\left(t^{0}\right) \Delta x_{r}^{i}\left(t^{0}\right)\right] \\
& +\limsup _{r \rightarrow \infty} k_{r}^{-2} \lambda_{\gamma} \Delta I_{\gamma}\left(\boldsymbol{a}_{r}\right)+\limsup _{r \rightarrow \infty} k_{r}^{-2} \mu_{\alpha}\left(t^{1}\right) \Delta \psi^{\alpha}\left(t^{1}, x_{r}\left(t^{1}\right)\right) \\
& \geqq \limsup _{r \rightarrow \infty} k_{r}^{-2} E_{T}\left(\boldsymbol{a}_{r}\right) .
\end{aligned}
$$

Using the inequality (128) we now obtain an important relation which will aid us in proving the admissibility of the variation $\eta_{0}$.

LEMMA 8.4.

$$
\lim _{r \rightarrow \infty} k_{r}^{-1} E_{T}\left(\boldsymbol{a}_{r}\right)=0
$$


Proof. The first three terms in (128) are bounded quantities. Also by the last item in (4-1) we have that

$$
\mu_{\alpha}\left(t^{0}\right) \leqq K^{\alpha} \quad \text { and } \quad \mu_{\alpha}\left(t^{0}\right)=K^{\alpha} \quad \text { if } \quad \psi^{\alpha}\left(t^{0}\right)<0 .
$$

Then by (130) together with the admissibility of our arcs which implies that for $r$ large enough

$$
\psi_{x^{i}}^{\alpha}\left(t^{0}\right) \Delta x_{r}^{i}\left(t^{0}\right) \leqq 0 \quad \text { if } \quad \psi^{\alpha}\left(t^{0}\right)=0
$$

we have for each $\alpha$ that

$$
-\left(\mu_{\alpha}\left(t^{0}\right)-K^{\alpha}\right) \psi_{x}^{\alpha}\left(t^{0}\right) \Delta x_{r}^{i}\left(t^{0}\right) \leqq 0 \quad(\alpha \text { not summed })
$$

Then by summing, multiplying by $k_{r}^{-2}$ and taking the superior limit we get

$$
\limsup _{r \rightarrow \infty} k_{r}^{-2}\left[-\left(\mu_{\alpha}\left(t^{0}\right)-K^{\alpha}\right) \psi_{x^{(}}^{\alpha}\left(t^{0}\right) \Delta x_{r}^{i}\left(t^{0}\right)\right] \leqq 0
$$

Furthermore by the definition (4-1) of the terms $\lambda_{\gamma}$ together with the admissibility of our arcs we see also that

$$
\limsup _{r \rightarrow \infty} k_{r}^{-2} \lambda_{\gamma} \Delta I_{\gamma}\left(a_{r}\right) \leqq 0
$$

and by the properties of $\mu_{\alpha}(t)$ as listed in (4) and again the admissibility of our arcs, also that

$$
\limsup _{r \rightarrow \infty} k_{r}^{-2} \mu_{\alpha}\left(t^{1}\right) \Delta \psi^{\alpha}\left(t^{1}, x_{r}\left(t^{1}\right)\right) \leqq 0
$$

Thus all terms on the left hand side of (128) are either bounded or non positive. Putting this statement together with the nonnegativity of $E_{T}\left(a_{r}\right)$ (which follows for large enough $r$ by (11)), then we get that

$$
\lim \sup k_{r}^{-2} E_{T}\left(a_{r}\right) \quad \text { is finite }
$$

thus proving (129) and the lemma.

By using Lemma 8.4 together with the break-up of functionals as in (109), we are now able to prove the analogue of Lemma 9.4 of [1] which we just state, since the proof is directly analagous to that used in [1].

LeMma 8.5. If $\mathscr{V}(t, x, p)$ is of class $C^{\prime}$ near $a_{0}$ and is $E_{T}$ dominated near $\boldsymbol{a}_{0}$ then 


$$
\lim _{r \rightarrow \infty} k_{r}^{-1}\left[\mathcal{V}\left(a_{r}, s\right)-\mathscr{V}\left(a_{0}, s\right)\right]=\mathscr{V}_{1}\left(\eta_{0}, s\right)
$$

by using (129) together with other observations obtained from (128), we are now in position to establish most of the requirements for admissibility of the variation $\eta_{0}$.

\section{Admissibility of the variation $\eta_{0}$.}

LEMMA 9.1. The variation $\eta_{0}$ of (101) satisfies the conditions (23-3), (23-5), (23-6), (23-7), (23-8), which are respectively

$$
\psi_{x}^{\alpha}\left(t^{1}\right) \eta_{0}^{t}\left(t^{1}\right)=0 \quad \text { if } \quad \mu_{\alpha}\left(t^{1}\right) \neq 0
$$

$$
\psi_{x^{\prime}}^{\alpha_{i}}\left(t^{0}\right) \eta_{0}^{i}\left(t^{0}\right)=0 \quad \text { if } \quad \mu_{\alpha}\left(t^{0}\right) \neq K^{\alpha}
$$

$$
J_{\gamma}^{\prime}\left(a_{0}, \eta_{0}\right)=0 \quad \text { if } \quad \lambda_{\gamma} \neq 0 \quad 1 \leqq \gamma \leqq p^{\prime}
$$

$$
\begin{aligned}
& J_{\gamma}^{\prime}\left(a_{0}, \eta_{0}\right) \leqq 0 \quad \text { if } \quad \lambda_{\gamma}=0 \quad \gamma \neq \gamma_{k} \quad 1 \leqq \gamma \leqq p^{\prime} \\
& J_{\rho}^{\prime}\left(a_{0}, \eta_{0}\right)=0 \quad p^{\prime}<\rho \leqq p+2 N
\end{aligned}
$$

where $\gamma_{k}$ are the indices of (22).

Proof. According to the statements used in proving Lemma 8.4 concerning the boundedness or the signs of terms in (128) we see in particular that

(138-1) $\lim \sup k_{r}^{-2} \mu_{\alpha}\left(t^{1}\right) \Delta \psi^{\alpha}\left(t^{1}, x_{r}\left(t^{1}\right)\right)$

is finite

(138-2) $\quad \limsup _{r \rightarrow \infty} k_{r}^{-2}\left[-\left(\mu_{\alpha}\left(t^{0}\right)-K^{\alpha}\right) \psi_{x}^{\alpha}\left(t^{0}\right) \Delta x_{r}^{i}\left(t^{0}\right)\right] \quad$ is finite and

(138-3) $\lim \sup k_{r}^{-2} \lambda_{\gamma} \Delta I_{\gamma}\left(a_{r}\right)$ is finite.

Now by an application of Taylor's Theorem together with the convergence of $\eta_{r}$ to $\eta_{0}$ we see that for each $\alpha$

(139-1) $\lim _{r \rightarrow \infty} k_{r}^{-1} \mu_{\alpha}\left(t^{1}\right) \Delta \psi^{\alpha}\left(t^{1}, x_{r}\left(t^{1}\right)\right)=\mu_{\alpha}\left(t^{1}\right) \psi_{x}^{\alpha}\left(t^{1}\right) \eta_{0}^{i}\left(t^{1}\right)$ ( $\alpha$ not summed).

Thus this limit exists for each $\alpha$. By summing on $\alpha$, we have that the sum of the limits exists and that by (138-1) this sum must vanish, that is

(139-2) $0=\lim _{r \rightarrow \infty} k_{r}^{-1} \mu_{\alpha}\left(t^{1}\right) \Delta \psi^{\alpha}\left(t^{1}, x_{r}\left(t^{1}\right)\right)=\mu_{\alpha}\left(t^{1}\right) \psi_{x^{i}}^{\alpha}\left(t^{1}\right) \eta_{0}^{i}\left(t^{1}\right)$. 
However by reasoning as used in obtaining (134), for each $\alpha$ index the product of the terms on the left side of (139-1) and hence also on the right side, is nonpositive. Putting this statement together with (139-2) establishes (137-1).

Next, we see by (15) that, $L_{\gamma}$ is $E_{T}$ dominated near $a_{0}$ so that by using Lemma 8.5 with $\mathscr{V}=L_{\gamma}$ we see that for each $\gamma$

$$
\begin{aligned}
\lim _{r \rightarrow \infty} k_{r}^{-1} \lambda_{\gamma} \Delta I_{\gamma}\left(a_{r}\right) & =\lambda_{\gamma}\left[g_{\gamma_{b} \sigma} \beta_{0}^{\sigma}+\int_{t^{0}}^{t^{1}}\left[L_{\gamma_{x}} \eta_{0}^{i}+L_{\gamma_{p} h} \alpha_{0}^{h}\right] d t\right] \\
& =\lambda_{\gamma} J_{\gamma}^{\prime}\left(a_{0}, \eta_{0}\right) \quad(\gamma \text { not summed })
\end{aligned}
$$

where the last equality follows from (20). Thus this limit exists, for each $\gamma$. By summing on $\gamma$ and using (139-3) we see that the sum of the limits exists and must vanish, that is

$$
0=\lim _{r \rightarrow \infty} k_{r}^{-1} \lambda_{\gamma} \Delta I_{\gamma}\left(\boldsymbol{a}_{r}\right)=\lambda_{\gamma} J_{\gamma}^{\prime}\left(\boldsymbol{a}_{0}, \eta_{0}\right)
$$

By similar statements as below (139-2), we see that for each $\gamma$

$$
\lambda_{\gamma} J_{\gamma}^{\prime}\left(a_{0}, \eta_{0}\right)=0 \quad(\gamma \text { not summed })
$$

Then by using the properties of the terms $\lambda_{\gamma}$ together with (140) and the admissibility of our arcs we get that (137-3) and (137-4) and (137-5) hold for $p^{\prime}<\rho \leqq p$. The remainder of (137-5) follows from the admissibility of our arcs and the definition (20).

Finally we note that the limit

(143) $\lim _{r \rightarrow \infty} k_{r}^{-1}\left[-\left(\mu_{\alpha}\left(t^{0}\right)-K^{\alpha}\right) \psi_{x^{\prime}}^{\alpha}\left(t^{0}\right) \Delta x_{r}^{i}\left(t^{0}\right)\right]=-\left(\mu_{\alpha}\left(t^{0}\right)-K^{\alpha}\right) \psi_{x^{\alpha}}^{\alpha}\left(t^{0}\right) \eta_{0}^{i}\left(t^{0}\right)$

certainly exists and then by steps similar to the above, but using (138-2), we get that (137-2) and hence the lemma is proven.

In order to establish the admissibility of the variation $\eta_{0}$, it remains only to prove that properties (23-1), (23-2) and (23-4) are satisfied. The property (23-2) follows from Taylor's Theorem together with the admissibility of our arcs so that

$$
\psi_{x^{\prime}}^{\alpha}(t) \eta_{0}^{i}(t) \leqq 0 \quad \text { on } \quad S^{\alpha} \quad \alpha=1, \cdots, m
$$

The property (23-4) is proven in the following lemma:

LEMmA 9.2. The variation $\eta_{0}$ satisfies condition (23-4). 
Proof. By the properties ${ }^{11}$ of $\dot{\mu}_{\alpha}(t)$ and by (144) we see that for each $\alpha$

$$
\dot{\mu}_{\alpha}(t) \psi_{x^{\alpha}}^{\alpha}(t) \eta_{0}^{i}(t) \geqq 0 \quad t^{0} \leqq t \leqq t^{1} \quad(\alpha \text { not summed })
$$

so that we will prove the desired result if we prove that

$$
\int_{t^{0}}^{t^{1}} \dot{\mu}_{\alpha}(t) \psi_{x^{\alpha}}^{\alpha}(t) \eta_{0}^{i}(t) d t=0
$$

Now, using the functions $\bar{\psi}^{\alpha}$ of (3), the definition of $k_{r}$ in (69) and the admissibility of our arcs we see that

$$
\begin{aligned}
0 & \leqq k_{r}^{-1} \int_{t^{0}}^{t^{1}} \dot{\mu}_{\alpha} \psi^{\alpha}\left(t, x_{r}\right) d t=k_{r}^{-1} \int_{S^{\alpha}} \dot{\mu}_{\alpha} \psi^{\alpha}\left(t, x_{r}\right) d t \\
& =k_{r}^{-1} \int_{S^{\alpha}} \dot{\mu} \bar{\psi}_{\alpha}^{\alpha}\left(t, x_{r}\right) d t=k_{r}^{-1} \int_{t^{0}}^{t^{1}} \dot{\mu}_{\alpha} \bar{\psi}^{\alpha}\left(t, x_{r}\right) d t \leqq k_{r}^{-1} k_{r}^{2}=k_{r} .
\end{aligned}
$$

Thus

$$
\lim _{r \rightarrow \infty} k_{r}^{-1} \int_{t^{0}}^{t^{1}} \dot{\mu}_{\alpha} \psi^{\alpha}\left(t, x_{r}\right) d t=0
$$

Now by the properties of $\dot{\mu}_{\alpha}$, we also have for each $\alpha$

$$
\dot{\mu}_{\alpha}(t) \psi^{\alpha}(t)=0 \quad t^{0} \leqq t \leqq t^{1} \quad(\alpha \text { not summed })
$$

so that we may add this to (148) and then get by Taylor's Theorem

$$
\begin{aligned}
0 & =\lim _{r \rightarrow \infty} k_{r}^{-1} \int_{t^{0}}^{t^{1}} \dot{\mu}_{\alpha} \psi^{\alpha}\left(t, x_{r}\right) d t=\lim _{r \rightarrow \infty} k_{r}^{-1} \int_{t^{0}}^{t^{1}} \dot{\mu}_{\alpha}\left[\psi^{\alpha}\left(t, x_{r}\right)-\psi^{\alpha}(t)\right] d t \\
& =\lim _{r \rightarrow \infty} \int_{t^{0}}^{t^{1}} \dot{\mu}_{\alpha} \tilde{\psi}_{x}^{\alpha} \eta_{r}^{i}(t) d t=\int_{t^{0}}^{t^{1}} \dot{\mu}_{\alpha} \psi_{x^{\alpha}}^{\alpha}(t) \eta_{0}^{i}(t) d t
\end{aligned}
$$

where $\tilde{\psi}_{x^{i}}^{\alpha_{i}}$ indicates evaluation on the line segment $t, x_{0}(t)+\theta \Delta x_{r}(t)$ $0<\theta<1$ and where the last equality follows from the uniform convergence of $x_{r}$ to $x_{0}$ and $\eta_{r}$ to $\eta_{0}$. Thus the lemma is proven.

The last required property for the admissibility of $\eta_{0}$ is established in the following lemma: 
LEMMA 9.3. With $f$ as the functions of (1-1), then the variation $\eta_{0}$ with quantities $\eta_{0}(t), \alpha_{0}(t)$ satisfies condition (23-1), that is

$$
\dot{\eta}_{0}^{i}(t)=f_{x^{i}}^{i}(t) \eta_{0}^{j}(t)+f_{p^{k}}^{i}(t) \alpha_{0}^{k}(t) \quad \text { a.e. on }\left[t^{0}, t^{1}\right]
$$

Proof. By Taylor's Theorem together with our $\Delta$ convention

$$
\dot{x}_{r}(t)-\dot{x}_{0}(t)=f_{x^{i}}(t) \Delta x_{r}^{i}(t)+f_{p^{k}}(t) \Delta p_{r}^{k}(t)+R_{r}(t) \text { on }\left[t^{0}, t^{1}\right]
$$

where

$$
\begin{gathered}
R_{r}(t) \equiv \int_{0}^{1}(1-\theta)\left[f_{x^{i} x^{i}} \Delta x_{r}^{i} \Delta x_{r}^{j}+2 f_{x^{i} p^{k}} \Delta x_{r}^{i} \Delta p_{r}^{k}+f_{p^{h} p^{k}} \Delta p_{r}^{h} \Delta p_{r}^{k}\right] d \theta \\
i, j=1, \cdots, N, \quad h, k=1, \cdots, K
\end{gathered}
$$

with the arguments of the $f$ partials being at

$$
\left(t, x_{0}+\grave{\theta} \Delta x_{r}, p_{0}+\theta \Delta p_{r}\right)
$$

Now let $s$ be a set on which $p_{r}$ converges uniformly to $p_{0}$, then

$$
\int_{s}\left|\frac{R_{r}(t)}{k_{r}}\right| d t \leqq \epsilon_{r} \int_{s}\left[\left|\Delta x_{r}^{i} \eta_{r}^{i}\right|+\left|\Delta x_{r}^{i} \alpha_{r}^{k}\right|+\left|\Delta p_{r}^{h} \alpha_{r}^{k}\right|\right] d t
$$

where $\epsilon_{r}$ is a constant which bounds the mixed partials of $f$ in the integrand of (153) and exists since $x_{r}$ converges uniformly to $x_{0}$ and $p_{r}$ converges to $p_{0}$ uniformly on $s$.

By Lemma 5.2 the integrals $\int_{s} \alpha_{r} d t$ are uniformly (with respect to $r$ ) bounded. Then by using this fact together with: (i) the uniform convergence of $x_{r}$ to $x_{0}$, and $\eta_{r}$ to $\eta_{0}$, (ii) the uniform convergence of $p_{r}$ to $p_{0}$ on $s$ and (iii) the fact that $\lim _{r \rightarrow \infty} \epsilon_{r}=0$, we see that

$$
\lim _{r \rightarrow \infty} \int_{s} \frac{R_{r}(t)}{k_{r}} d t=0
$$

Thus by (139) and (152) we obtain

$$
\lim _{r \rightarrow \infty} \int_{s} \dot{\eta}_{r}^{\prime} d t=\lim _{r \rightarrow \infty} \int_{s}\left[f_{x^{i}}^{i} \eta_{r}^{j}+f_{p^{k}}^{i} \alpha_{r}^{k}\right] d t
$$

Now by Theorem 6.2 and 6.3 together with (157) we have 


$$
\begin{aligned}
\int_{s} \dot{\eta}_{0}^{i} d t & =\lim _{r \rightarrow \infty} \int_{s} \dot{\eta}_{r}^{i} d t=\lim _{r \rightarrow \infty} \int_{s}\left[f_{x}^{i} \eta_{r}^{j}+f_{p^{k}}^{i} \alpha_{r}^{k}\right] d t \\
& =\int_{s}\left[f_{x^{i}}^{i} \eta_{0}^{j}+f_{p^{k}}^{i} \alpha_{0}^{k}\right] d t
\end{aligned}
$$

so that on sets $s$ upon which $p_{r}$ converges uniformly to $p_{0}$, we have

$$
\int_{s} \dot{\eta}_{0}^{i} d t=\int_{s}\left[f_{x^{j}}^{i} \eta_{0}^{j}+f_{p^{k}}^{i} \alpha_{0}^{k}\right] d t
$$

Since $p_{r}$ converges [a.unif.] to $p_{0}$ and (159) holds on each such set where this convergence is uniform then for any set $M$

$$
\int_{M} \dot{\eta}_{0}^{i} d t=\int_{M}\left[f_{x}^{j} \eta_{0}^{j}+f_{p^{k}}^{i} \alpha_{0}^{k}\right] d t
$$

and the Theorem is proven.

Thus by Lemmas 9.1 through 9.3 together with (144) we see that the variation $\eta_{0}$ is admissible.

10. Proof of Theorem 3.1. We are now in position to prove Theorem 3.1 as follows:

THEOREM 10.1. If the following two inequalities are true, then Theorem 3.1 is true.

$$
\begin{aligned}
& \limsup _{r \rightarrow \infty} k_{r}^{-2} E_{T}\left(\boldsymbol{a}_{r}\right) \geqq \frac{1}{2} \int_{t^{0}}^{t^{1}} G_{p^{k} p^{h}}\left(\alpha_{0}^{k}-\rho_{0}^{k}\right)\left(\alpha_{0}^{h}-\rho_{0}^{h}\right) d t \quad h, k=1, \cdots, K . \\
& 0 \geqq \limsup _{r \rightarrow \infty} k_{r}^{-2}\left[-\left(\mu_{\alpha}\left(t^{0}\right)-K^{\alpha}\right) \psi_{x^{\alpha}}^{\alpha}\left(t^{0}\right) \Delta x_{r}^{i}\left(t^{0}\right)\right] \\
& \quad-\frac{1}{2}\left(\mu_{\alpha}\left(t^{0}\right)-K^{\alpha}\right) \psi_{x^{i} x}^{\alpha^{i}}\left(t^{0}\right) \eta_{0}^{i}\left(t^{0}\right) \eta_{0}^{j}\left(t^{0}\right) \quad i, j=1, \cdots, N
\end{aligned}
$$

(where the arguments of $G_{p^{k} p^{h}}$ are those of $a_{0}$ ).

Proof. Referring to inequality (128) and using arguments as used in the proof of Lemma 8.4, we see that the fifth and sixth terms of (128) are non positive. Now assuming the truth of the above listed inequalities, we see that (128) implies that

$$
J_{2}\left(a_{0}, \eta_{0}\right) \leqq 0
$$


which according to the hypotheses of Theorem 3.1, implies that the variation $\eta_{0}$ of (101) is null. According to the formula for $\rho_{0}$ as listed in (104), then this quantity is also null so that

$$
\int_{t^{0}}^{t^{1}} G_{p^{k} p^{h}}\left(\alpha_{0}^{h}-\rho_{0}^{h}\right)\left(\alpha_{0}^{k}-\rho_{0}^{k}\right) d t=0
$$

but then by (128) again, this implies that

$$
0 \geqq \limsup _{r \rightarrow \infty} k_{r}^{-2} E_{T}\left(\boldsymbol{a}_{r}\right)
$$

which by the nonnegativity of this quantity for large $r$, implies that

$$
0=\limsup _{r \rightarrow \infty} k_{r}^{-2} E_{T}\left(a_{r}\right)
$$

Next by reasoning similar to that used in [1] p. 47, we see that there is a positive number $b^{*}$ such that for $r$ large enough

$$
E_{L}\left(P_{r}, p_{r}\right) \geqq b^{*} E_{L}\left(P_{r}-p_{0}, p_{r}-p_{0}\right) .
$$

Then by (11), we get that for large $r$,

$$
\begin{aligned}
k_{r}^{-2} E_{T}\left(a_{r}\right) \geqq & b k_{r}^{-2}\left[\int_{t^{0}}^{t^{1}} E_{L}\left(P_{r}, p_{r}\right) d t\right. \\
& \left.+\max \left(\int_{t^{0}}^{t^{1}} \dot{\mu}_{\alpha} \bar{\psi}^{\alpha}\left(t, x_{r}\right) d t, \int_{t^{0}}^{t^{1}}\left|\dot{\mu}_{\alpha} \bar{\phi}^{\alpha}\left(t, x_{r}, p_{r}\right)\right| d t\right)\right] \\
\geqq & b b^{*} k_{r}^{-2}\left[\int_{t^{0}}^{t^{1}} E_{L}\left(P_{r}-p_{0}, p_{r}-p_{0}\right) d t\right. \\
& \left.+\max \left(\int_{t^{0}}^{t^{1}} \dot{\mu}_{\alpha} \bar{\psi}^{\alpha}\left(t, x_{r}\right) d t, \int_{t^{0}}^{t^{1}}\left|\dot{\mu}_{\alpha} \bar{\phi}^{\alpha}\left(t, x_{r}, p_{r}\right)\right| d t\right)\right] \\
= & b b^{*} k_{r}^{-2}\left[\int_{t^{0}}^{t^{1}}\left[L\left(p_{r}-p_{0}\right)-\frac{\left.1+k_{r}^{2} \rho_{r}^{k} \alpha_{r}^{k}\right]}{L\left(P_{r}-p_{0}\right)}\right] d t\right. \\
& \left.+\dot{m a x}\left(\int_{t^{0}}^{t^{1}} \dot{\mu}_{\alpha} \bar{\psi}^{\alpha}\left(t, x_{r}\right) d t, \int_{t^{0}}^{t^{1}}\left|\dot{\mu}_{\alpha} \bar{\phi}^{\alpha}\left(t, x_{r}, p_{r}\right)\right| d t\right)\right] \\
\geqq & b b^{*} k_{r}^{-2}\left[\int_{t^{0}}^{t^{1}}\left[L\left(p_{r}-p_{0}\right)-1-\frac{k_{r}^{2} \rho_{r}^{k} \alpha_{r}^{k}}{L\left(P_{r}-p_{0}\right)}\right] d t\right. \\
& \left.+\max \left(\int_{t^{0}}^{t^{1}} \dot{\mu}_{\alpha} \bar{\psi}^{\alpha}\left(t, x_{r}\right) d t, \int_{t^{0}}^{t^{1}}\left|\dot{\mu}_{\alpha} \bar{\phi}^{\alpha}\left(t, x_{r}, p_{r}\right)\right| d t\right)\right] .
\end{aligned}
$$


By Lemma 5.2 together with the fact that the variation $\eta_{0}$ is null so that $\rho_{r}$ converges uniformly to zero, $\beta_{0}^{2}=0, \max _{\left[t^{0}, t^{1}\right]}\left|\eta_{0}(t)\right|^{2}=0$, and by taking superior limits of (166), we obtain:

$$
\begin{aligned}
(167) \limsup _{r \rightarrow \infty} k_{r}^{-2} E_{T}\left(\boldsymbol{a}_{r}\right) \geqq & \limsup _{r \rightarrow \infty} b b^{*} k_{r}^{-2}\left[K\left(\boldsymbol{a}_{r}, \boldsymbol{a}_{0}\right)\right. \\
& +\max \left(\int_{t^{0}}^{t^{1}} \dot{\mu}_{\alpha} \bar{\psi}^{\alpha}\left(t, x_{r}\right) d t, \int_{t^{0}}^{t^{1}}\left|\dot{\mu}_{\alpha} \bar{\phi}^{\alpha}\left(t, x_{r}, p_{r}\right)\right| d t\right) \\
& \left.+\left|\beta_{r}\right|^{2}+\max _{\left[t^{0}, t^{1}\right]}\left|\eta_{r}(t)\right|^{2}\right]=b b^{*}>0
\end{aligned}
$$

which is a contradiction, thus proving the theorem and hence also Theorem 3.1.

We now prove the second of the inequalities listed in Theorem 10.1.

LEMMA 10.1. The following inequality is true

$$
\begin{aligned}
& 0 \geqq \lim \sup k_{r}^{-2}\left[-\left(\mu_{\alpha}\left(t^{0}\right)-K^{\alpha}\right) \psi_{x^{\prime}}^{\alpha}\left(t^{0}\right) \Delta x_{r}^{i}\left(t^{0}\right)\right] \\
& -\frac{1}{2}\left(\mu_{\alpha}\left(t^{0}\right)-K^{\alpha}\right) \psi_{x^{\prime} x^{\prime}}^{\alpha}\left(t^{0}\right) \eta_{0}^{t}\left(t^{0}\right) \eta_{0}^{\prime}\left(t^{0}\right) . \\
& i, j=1, \cdots, N \quad \alpha=1, \cdots, m .
\end{aligned}
$$

Proof. According to the last property in (4-1), we see that we need only consider those indices $\bar{\alpha}$ such that

$$
\psi^{\bar{\alpha}}\left(t^{0}\right)=0
$$

Then by Taylor's theorem together with the admissibility of our arcs, we have that for each such $\bar{\alpha}$

$$
\begin{aligned}
& 0 \geqq \Delta \psi^{\bar{\alpha}}\left(t^{0}, x_{r}\left(t^{0}\right)\right)=\psi_{x}^{\bar{\alpha}} \cdot\left(t^{0}\right) \Delta x_{r}^{i}\left(t^{0}\right) \\
& +\frac{1}{2} \tilde{\psi}_{x^{\prime} x^{\prime}}^{\bar{\alpha}} \Delta x_{r}^{i}\left(t^{0}\right) \Delta x_{r}^{j}\left(t^{0}\right) \quad i, j=1, \cdots, N
\end{aligned}
$$

where $\tilde{\psi}_{x}^{\bar{\alpha}}{ }^{\prime} x^{\prime}$ indicates evaluation at an intermediate point on the line segment $\left[x_{0}\left(t^{0}\right)+\theta \Delta x_{r}\left(t^{0}\right)\right] \quad 0<\theta<1$. Now by multiplying by $-k_{r}^{-2}\left(\mu_{\bar{\alpha}}\left(t^{0}\right)-K^{\bar{\alpha}}\right)$, taking superior limits, using the convergence of $\eta_{r}\left(t^{0}\right)$ to $\eta_{0}\left(t^{0}\right)$ and the last property in (4-1), we get (168) for each such index $\bar{\alpha}$ and hence for the sum of those indices, proving the lemma.

It remains only to prove the first inequality listed in the hypothesis of Theorem 10.1. By using arguments directly analagous to those used in 
Lemma 11.3 of [1], but with $\dot{\mu}_{\alpha}$ replacing $\lambda^{\beta}$, one proves the required inequality with lim inf replacing lim sup. Since lim sup $\geqq \lim$ inf, the required inequality is certainly true. We state this result

LEMMA 10.2. The following inequality is true

$$
\begin{aligned}
\lim \sup k_{r}^{-2} E_{T}\left(\boldsymbol{a}_{r}\right) \geqq \frac{1}{2} \int_{t^{0}}^{t^{1}} G_{p^{h} p^{k}}\left(\alpha_{0}^{h}-\rho_{0}^{h}\right)\left(\alpha_{0}^{k}-\rho_{0}^{k}\right) d t & \\
& h, k=1, \cdots, K .
\end{aligned}
$$

\section{REFERENCES}

1. L. L. Pennisi, An Indirect Sufficiency Proof For the Problem of Lagrange With Differential Inequalities as Side Conditions, Dissertation at University of Chicago, 1952.

2. I. B. Russak, Second order necessary conditions for problems with state inequality constraints SIAM J. Control, 13, No. 2, (1975).

3. On problems with bounded state variables, J. Optimization Theory and Appl., 5 No. 2, (1970).

Received June 10, 1975. Supported by an NPS Foundation grant.

Naval Postgraduate School-Monterey 




\section{Pacific Journal of Mathematics}

\section{Vol. 62, No. $1 \quad$ January, 1976}

Mieczyslaw Altman, Contractor directions, directional contractors and

directional contractions for solving equations . .................. 1

Michael Peter Anderson, Subgroups of finite index in profinite groups .........

Zvi Arad, Abelian and nilpotent subgroups of maximal order of groups of odd order

John David Baildon and Ruth Silverman, On starshaped sets and Helly-type theorems ..........................................

John W. Baker and R. C. Lacher, Some mappings which do not admit an

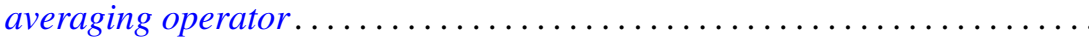

Joseph Barback, Composite numbers and prime regressive isols . . . . . . . . . .

David M. Boyd, Composition operators on $H^{p}(A) \ldots \ldots \ldots \ldots \ldots \ldots \ldots$

Maurice Chacron, Co-radical extension of PI rings . . . . . . . . . . . . .

Fred D. Crary, Some new engulfing theorems . . . . . . . . . . . . . . .

Victor Dannon and Dany Leviatan, A representation theorem for convolution transform with determining function in $L^{p} \ldots \ldots \ldots \ldots \ldots \ldots \ldots \ldots \ldots \ldots \ldots \ldots \ldots \ldots$

Mahlon M. Day, Lumpy subsets in left-amenable locally compact

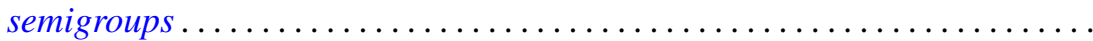

Michael A. Gauger, Some remarks on the center of the universal enveloping algebra of a classical simple Lie algebra . .

David K. Haley, Equational compactness and compact topologies in rings

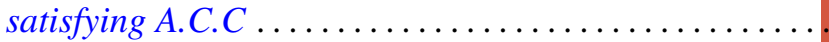

Raymond Heitmann, Generating ideals in Prüfer domains .

Gerald Norman Hile, Entire solutions of linear elliptic equations with

Laplacian principal part. .

Richard Oscar Hill, Moore-Postnikov towers for fibrations in which $\pi_{1}$ (fiber) is non-abelian

John Rast Hubbard, Approximation of compact homogeneous maps . .

Russell L. Merris, Relations among generalized matrix functions . .

V. S. Ramamurthi and Edgar Andrews Rutter, On cotorsion radicals ...

Ralph Tyrrell Rockafellar and Roger Jean-Baptiste Robert Wets, Stochastic

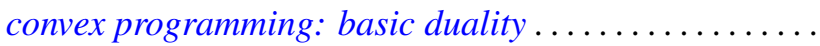

Alban J. Roques, Local evolution systems in general Banach spaces ..

I. Bert Russak, An indirect sufficiency proof for problems with bounded state variables.

Richard Alexander Sanerib, Jr., Ultrafilters and the basis property. .

H. A. Seid, The decomposition of multiplication operators on $L_{p}$-spaces . .

Franklin D. Tall, The density topology .................. 